TRANSACTIONS OF THE

AMERICAN MATHEMATICAL SOCIETY

Volume 355, Number 3, Pages 1145-1165

S 0002-9947(02)03175-6

Article electronically published on November 5, 2002

\title{
SINGULAR INTEGRALS WITH ROUGH KERNELS ALONG REAL-ANALYTIC SUBMANIFOLDS IN $\mathbf{R}^{3}$
}

\author{
DASHAN FAN, KANGHUI GUO, AND YIBIAO PAN
}

\begin{abstract}
L^{p}$ mapping properties will be established in this paper for singular Radon transforms with rough kernels defined by translates of a real-analytic submanifold in $\mathbf{R}^{3}$.
\end{abstract}

\section{INTRODUCTION}

The main purpose of this paper is to establish the $L^{p}$ boundedness of singular integral operators with rough kernels supported by real-analytic submanifolds in $\mathbf{R}^{3}$. For $y \in \mathbf{R}^{n}$, let $K(y)$ be a Calderón-Zygmund type kernel of the form

$$
K(y)=b(|y|) \frac{\Omega(y)}{|y|^{n}},
$$

where $\Omega$ is homogeneous of degree 0 , integrable over $\mathbf{S}^{n-1}$ and satisfies

$$
\int_{\mathbf{S}^{n-1}} \Omega(y) d \sigma(y)=0,
$$

and $b:[0, \infty) \rightarrow \mathbf{C}$ is a measurable function. Let $d \in \mathbf{N}$ and let $\mathbf{U}$ be a neighborhood of the origin in $\mathbf{R}^{n}$. For a suitable mapping $\boldsymbol{\Psi}: \mathbf{U} \rightarrow \mathbf{R}^{d}$ we define the singular integral operator $T_{\boldsymbol{\Psi}}$ on $\mathbf{R}^{d}$ by

$$
\left(T_{\boldsymbol{\Psi}} f\right)(x)=\mathrm{p} \cdot \mathrm{v} \cdot \int_{\mathbf{U}} f(x-\mathbf{\Psi}(y)) K(y) d y .
$$

When $n=d, \mathbf{U}=\mathbf{R}^{n}$ and $\boldsymbol{\Psi}(y) \equiv y, T_{\boldsymbol{\Psi}}$ becomes the classical singular integral operator $T_{I}$ :

$$
\left(T_{I} f\right)(x)=\mathrm{p} \cdot \mathrm{v} \cdot \int_{\mathbf{R}^{n}} f(x-y) K(y) d y .
$$

By introducing the "method of rotations", Calderón and Zygmund proved in 1956 that the operator $T_{I}$ is bounded on $L^{p}\left(\mathbf{R}^{n}\right)$ for $1<p<\infty$ when $b \equiv 1$ and $\Omega \in L \log L\left(\mathbf{S}^{n-1}\right)$. If we let $H^{1}\left(\mathbf{S}^{n-1}\right)$ denote the Hardy space on the unit sphere, then $L \log L\left(\mathbf{S}^{n-1}\right) \subset H^{1}\left(\mathbf{S}^{n-1}\right) \subset L^{1}\left(\mathbf{S}^{n-1}\right)$ and the method of Calderón and Zygmund allows the condition $\Omega \in L \log L\left(\mathbf{S}^{n-1}\right)$ to be weakened to $\Omega \in$ $H^{1}\left(\mathbf{S}^{n-1}\right)$ for the $L^{p}$ boundedness of $T_{I}$ with $b \equiv 1$ (see [3], 20], [5]). On the

Received by the editors March 16, 1998 and, in revised form, July 14, 2002.

2000 Mathematics Subject Classification. Primary 42B20; Secondary 42B15, $42 \mathrm{~B} 25$.

Key words and phrases. Singular integrals, oscillatory integrals, Fourier transform, maximal functions, $L^{p}$ boundedness, rough kernels, real-analytic submanifolds.

This work was done during the second author's visit at the Department of Mathematics, University of Pittsburgh.

The third author was partially supported by NSF Grant DMS-9622979. 
other hand, Calderón and Zygmund showed that the $L^{p}$ boundedness of $T_{I}$ may fail if the condition $\Omega \in L \log L\left(\mathbf{S}^{n-1}\right)$ is replaced by any weaker metric condition $\Omega \in L^{\phi}\left(\mathbf{S}^{n-1}\right)$ with a $\phi$ satisfying $\phi(t)=o(t \log t)$ as $t \rightarrow \infty$ (e.g., $L^{1}=L^{\phi}$ with $\phi(t)=t)$.

The study of the operator $T_{I}$ with a nonsmooth factor $b(|y|)$ in its kernel was initiated by R. Fefferman in 8 and continued by many authors. It is now known that the operator $T_{I}$ is bounded on $L^{p}\left(\mathbf{R}^{n}\right)$ for $1<p<\infty$ when $\Omega \in H^{1}\left(\mathbf{S}^{n-1}\right)$ and $b$ satisfies a very mild condition (see the condition (1.6) below).

For a general mapping $\boldsymbol{\Psi}$, the operator $T_{\boldsymbol{\Psi}}$ belongs to the class of singular Radon transforms whose $L^{p}$ mapping properties are relatively well understood when the Calderón-Zygmund kernel $K(y)$ is smooth away from the origin. To understand the importance of these operators and recent advances in this area we refer the reader to Stein's survey paper 21] and his book [23, and the papers [1, [2, [13, 16]-19].

The primary focus of our investigation is the $L^{p}$ boundedness of the operator $T_{\boldsymbol{\Psi}}$ when $\boldsymbol{\Psi}$ is a general mapping and $K$ is allowed to be nonsmooth on the unit sphere as well as in the radial direction. In the paper by the first and third authors ([9]), the $L^{p}$ boundedness of $T_{\boldsymbol{\Psi}}$ was established for all $\boldsymbol{\Psi}$ defined by polynomial functions on $\mathbf{R}^{n}$. Below is a theorem from [9].

Theorem $1.1(9])$. Let $d, n \in \mathbf{N}, n \geq 2, x \in \mathbf{U}=\mathbf{R}^{n}$ and $\mathcal{P}(x)=\left(P_{1}(x), \ldots\right.$, $\left.P_{d}(x)\right)$ with $P_{j}(\cdot)$ being polynomials with real coefficients. Let $T_{\mathcal{P}}$ be defined by (1.1)-(1.3). Suppose that

$$
\Omega \in H^{1}\left(\mathbf{S}^{n-1}\right)
$$

and

$$
\sup _{R>0} \frac{1}{R} \int_{0}^{R}|b(t)|^{\gamma} d t<\infty, \text { for some } \gamma>1 .
$$

Then for $\left|\frac{1}{p}-\frac{1}{2}\right|<\min \left\{\frac{1}{2}, \frac{1}{\gamma^{\prime}}\right\}$ there exists a constant $C_{p}>0$ such that

$$
\left\|T_{\mathcal{P}} f\right\|_{L^{p}\left(\mathbf{R}^{d}\right)} \leq C_{p}\|\Omega\|_{H^{1}\left(\mathbf{S}^{n-1}\right)}\|f\|_{L^{p}\left(\mathbf{R}^{d}\right)} .
$$

The constant $C_{p}$ may depend on $p, d, n, b(\cdot)$ and $\operatorname{deg}\left(P_{j}\right)$, but it is independent of the coefficients of the $P_{j}$ 's.

The natural question is how to extend Theorem 1.1 from the submanifolds given by polynomials to the ones given by real-analytic functions in $R^{n}$. In a recent paper $([10])$, we realized that if one replaces the $H^{1}$ condition (1.5) for $\Omega$ with the condition $\Omega \in L^{q}\left(\mathbf{S}^{n-1}\right), q>1$, then the extension is relatively easy. Examining the proof of Theorem 1.1, one will see that the key idea is to reduce a polynomial of order $m$ to a polynomial of order $m-1$, then to $m-2$ and so on. This argument obviously breaks down for real-analytic functions since in general no highest-order term(s) can be found for such functions; so new ideas are imperative if one does not wish to strengthen condition (1.5) for real-analytic submanifolds. It turns out that we need to obtain various oscillatory integral estimates which are essentially different from those developed in [9]. At the present point, we are able to get what we want only for the case $n=2$ and $d=3$ due to the fact that one of the oscillatory integral estimates works only for this case (see Theorem 3.7).

Also, we want to point out that even though condition (1.6) is weaker than the condition $b \in L^{\infty}$, the proof of Theorem 1.1 in [9] and the proof of Theorem 1.2 in [10] are only technically different for both conditions. In order to avoid some 
confusion of the notation, in this paper we present our following result with the condition $b \in L^{\infty}$ even though it holds under condition (1.6) (with a $p$ dependent on $\gamma$ as stated in (1.7)).

Theorem 1.2. Let $\mathbf{U}$ be a bounded neighborhood of the origin in $\mathbf{R}^{2}$ and $\boldsymbol{\Psi}$ a realanalytic mapping from $\mathbf{U}$ into $\mathbf{R}^{3}$ given by $\mathbf{\Psi}(w)=(w, \psi(w))$. Let $T_{\boldsymbol{\Psi}}$ be defined by (1.1)-(1.3). If $\Omega$ satisfies (1.5) and $b(\cdot) \in L^{\infty}$, then there exists a positive constant $C_{p}=C(p, b, \boldsymbol{\Psi})$ such that

$$
\left\|T_{\mathbf{\Psi}} f\right\|_{L^{p}\left(\mathbf{R}^{3}\right)} \leq C_{p}\|\Omega\|_{H^{1}\left(\mathbf{S}^{1}\right)}\|f\|_{L^{p}\left(\mathbf{R}^{3}\right)}, \quad 1<p<\infty .
$$

The paper is organized as follows. A reduction lemma on maximal functions is given in section 2. Section 3 contains several oscillatory integral estimates including a variation of van der Corput's lemma (Lemma 3.3) which, in addition to being a key element in our proofs, may have applications elsewhere. In section 4 we use the reduction lemma obtained in section 2 to establish a key estimate of maximal functions (Theorem 4.7), from which Theorem 1.2 will be derived.

The authors would like to thank the referee for his helpful criticism and suggestions.

\section{A Reduction lemma on maximal functions}

For a sequence of measures $\left\{\sigma_{k}\right\}_{k \in \mathbf{Z}}$ on $\mathbf{R}^{d}$ we define the operator $\sigma^{*}$ by

$$
\sigma^{*}(f)(x)=\sup _{k \in \mathbf{Z}}|| \sigma_{k}|* f(x)| .
$$

For $b(\cdot) \in L^{\infty}, \Omega(\cdot) \in L^{1}\left(S^{n-1}\right)$, let $K(w)$ be given by (1.1). For $k \in \mathbf{Z}_{-}$we define $D_{k} \subset \mathbf{R}^{n}$ by

$$
D_{k}=\left\{w \in \mathbf{R}^{n}\left|2^{k} \leq\right| w \mid<2^{k+1}\right\} .
$$

For a smooth mapping $\Gamma: \mathbf{R}^{n} \rightarrow \mathbf{R}^{d}$ we define the measures $\sigma_{k, \Gamma}$ on $\mathbf{R}^{d}$ by

$$
\int_{\mathbf{R}^{d}} f d \sigma_{k, \Gamma}=\int_{D_{k}} f(\Gamma(w)) K(w) d w
$$

We also introduce positive measures $\mu_{k, \Gamma}$ :

$$
\int_{\mathbf{R}^{d}} f d \mu_{k, \Gamma}=\int_{D_{k}} f(\Gamma(x))|K(w)| d w .
$$

For the Fourier transforms of $\sigma_{k, \Gamma}$ and $\mu_{k, \Gamma}$, we have

$$
\begin{gathered}
\hat{\sigma}_{k, \Gamma}(\xi)=\int_{2^{k}}^{2^{k+1}} \int_{S^{n-1}} e^{i \xi \cdot \Gamma(t w)} \Omega(w) d \sigma(w) \frac{b(t)}{t} d t, \\
\hat{\mu}_{k, \Gamma}(\xi)=\int_{2^{k}}^{2^{k+1}} \int_{S^{n-1}} e^{i \xi \cdot \Gamma(t w)}|\Omega(w)| d \sigma(w) \frac{|b(t)|}{t} d t .
\end{gathered}
$$

Also, since $\Omega \in L^{1}\left(S^{n-1}\right)$ and $b \in \infty$, we see that $\left|\hat{\mu}_{k, \Gamma}(\xi)\right| \leq C$ for all $\xi \in \mathbf{R}^{d}$ and $k \in \mathbf{Z}_{-}$. For a measurable function $f$ on $\mathbf{R}^{d}$ we define $\mu_{\Gamma}^{*}(f)$ by

$$
\mu_{\Gamma}^{*}(f)(y)=\sup _{k \in \mathbf{Z}_{-}}\left|\left(\mu_{k, \Gamma} * f\right)(y)\right| .
$$

We call $\mu_{\Gamma}^{*}$ the maximal function associated to $\left\{\mu_{k, \Gamma}\right\}$. 
Lemma 2.1. Let $\xi \in \mathbf{R}^{d}, a_{0}>1,0<\alpha_{0}<1, N>0$ and $T_{0}: \mathbf{R}^{d} \rightarrow \mathbf{R}^{m}$ be a linear transformation and let $\Gamma_{0}, \Gamma_{1}: \mathbf{R}^{n} \rightarrow \mathbf{R}^{d}$ be smooth mappings. If

$$
\begin{gathered}
\left|\hat{\mu}_{k, \Gamma_{0}}(\xi)\right| \leq A_{0}\left(a_{0}^{k}\left|T_{0} \xi\right|\right)^{-\alpha_{0}}, \\
\left|\hat{\mu}_{k, \Gamma_{0}}(\xi)-\hat{\mu}_{k, \Gamma_{1}}(\xi)\right| \leq A_{1} a_{0}^{k}\left|T_{0} \xi\right|, \\
\left\|\mu_{\Gamma_{1}}^{*} f\right\|_{p} \leq B_{p}\|f\|_{p}, \quad 1<p<\infty
\end{gathered}
$$

for $\xi \in \mathbf{R}^{d}, k \in \mathbf{Z}_{-},|k|>N$ and $f \in L^{p}\left(\mathbf{R}^{d}\right)$, then we have

$$
\left\|\mu_{\Gamma_{0}}^{*} f\right\|_{p} \leq C_{p}\|f\|_{p}, \quad 1<p<\infty
$$

for $f \in L^{p}\left(\mathbf{R}^{d}\right)$, where $C_{p}$ may depend on $A_{0}, A_{1}, N, B_{p}, \alpha_{0}$ and $a_{0}$, but is independent of the linear transformation $T_{0}$.

Proof. Let $s=\operatorname{rank}\left(T_{0}\right)$ and $\pi_{s}^{d}$ be the projection operator from $\mathbf{R}^{d}$ to $\mathbf{R}^{s}$. Using Lemma 6.1 in [9], one can find a nonsingular linear transformation $G: \mathbf{R}^{d} \rightarrow \mathbf{R}^{d}$ such that

$$
\left|\pi_{s}^{d} G \xi\right| \leq\left|T_{0} \xi\right| \leq(m-s+1)\left|\pi_{s}^{d} G \xi\right|
$$

for every $\xi \in \mathbf{R}^{d}$.

Let $S\left(\mathbf{R}^{s}\right)$ be the Schwartz class functions in $\mathbf{R}^{s}$. We choose and fix a function $\varphi \in C_{0}^{\infty}\left(\mathbf{R}^{s}\right)$ be such that $\varphi(t) \equiv 1$ for $|t| \leq 1 / 2$, and $\varphi(t) \equiv 0$ for $|t| \geq 1$. Let $\Phi \in \mathcal{S}\left(\mathbf{R}^{s}\right)$ such that $\hat{\Phi}=\varphi$. Define $J$ and $X_{r}=X_{r}(\varphi, G)$ by $(J f)(y)=f\left(G^{t} y\right)$ and

$$
X_{r} f(y)=J^{-1}\left(\left(\left|\Phi_{r}\right| \otimes \delta_{\mathbf{R}^{d-s}}\right) * J f\right)(y),
$$

where $r>0$ and $\Phi_{r}(x)=\frac{1}{r^{s}} \Phi\left(\frac{1}{r} x\right), x \in \mathbf{R}^{s}$. Let $X=X(\varphi, G)$ be given by

$$
X f(y)=\sup _{r>0}\left|X_{r} f(y)\right| .
$$

It is easy to show (see Lemma 6.4 in [9]) that for $1<p<\infty$ there exists a positive constant $C_{p}=C(p, \varphi, s, d)$ such that

$$
\|X f\|_{p} \leq C_{p}\|f\|_{p}
$$

for $f \in L^{p}\left(\mathbf{R}^{d}\right)$. The fact that the constant $C_{p}$ is independent of the linear transformation $G$ is crucial for the latter application of Lemma 2.1.

For $k \in \mathbf{Z}_{-}$we define the measures $\nu_{k}$ and $\tau_{k}$ by

$$
\hat{\nu}_{k}(\xi)=\varphi\left(a_{0}^{k} \pi_{s}^{d} G \xi\right) \hat{\mu}_{k, \Gamma_{1}}(\xi)
$$

and

$$
\tau_{k}=\mu_{k, \Gamma_{0}}-\nu_{k}
$$

We also set

$$
\tau^{*}(f)(y)=\sup _{k \in \mathbf{Z}_{-}}\left|\left(\left|\tau_{k}\right| * f\right)(y)\right|
$$

and

$$
\nu^{*}(f)(y)=\sup _{k \in \mathbf{Z}_{-}}\left|\left(\left|\nu_{k}\right| * f\right)(y)\right| .
$$

Since

$$
\left(\nu_{k} * f\right)(y)=J^{-1}\left[\left(\Phi_{a_{0}^{k}} \otimes \delta_{\mathbf{R}^{d-s}}\right) * J\left(\mu_{k, \Gamma_{1}} * f\right)\right](y)
$$


and

$$
\left|\nu_{k}\right| * f(y) \leq X_{a_{0}^{k}}\left(\mu_{k, \Gamma_{1}} *|f|\right)(y)
$$

we have

$$
\nu^{*}(f)(y) \leq X\left(\mu_{\Gamma_{1}}^{*}(|f|)\right)(y) .
$$

Then (2.6), (2.11) and (2.18) give

$$
\left\|\nu^{*}(f)\right\|_{p} \leq C_{p}\|f\|_{p}, \quad 1<p<\infty .
$$

We now follow the bootstrap argument in [6] (see also [9]).

By $\varphi(0)=1,(2.5)$ and $(2.8)$ we have

$$
\begin{aligned}
\left|\hat{\tau}_{k}(\xi)\right| & \leq\left|\hat{\mu}_{k, \Gamma_{0}}(\xi)-\hat{\mu}_{k, \Gamma_{1}}(\xi)\right|+\left|1-\varphi\left(a_{0}^{k} \pi_{s}^{d} G \xi\right)\right|\left|\hat{\mu}_{k, \Gamma_{1}}(\xi)\right| \\
& \leq C a_{0}^{k}\left|T_{0} \xi\right|
\end{aligned}
$$

for $k \in \mathbf{Z}_{-}$and $\xi \in \mathbf{R}^{d}$. (2.4), (2.8), and (2.12) imply that

$$
\left|\hat{\tau}_{k}(\xi)\right| \leq C\left(a_{0}^{k}\left|T_{0} \xi\right|\right)^{-\alpha_{0}}
$$

whenever $\left|T_{0} \xi\right| \geq m\left(a_{0}^{k}\right)^{-1}$. Combining (2.20) and (2.21) we find

$$
\left|\hat{\tau}_{k}(\xi)\right| \leq C\left[\min \left\{a_{0}^{k}\left|T_{0} \xi\right|,\left(a_{0}^{k}\left|T_{0} \xi\right|\right)^{-1}\right\}\right]^{\alpha_{0}}
$$

for $k \in \mathbf{Z}_{-}$and $\xi \in \mathbf{R}^{d}$. Set

$$
g_{\Gamma_{0}}(f)(y)=\left(\sum_{k \in \mathbf{Z}_{-}}\left|\tau_{k} * f(y)\right|^{2}\right)^{\frac{1}{2}} .
$$

Then by (2.13) we have

$$
\mu_{\Gamma_{0}}^{*}(f)(y) \leq g_{\Gamma_{0}}(f)(y)+\nu^{*}(f)(y)
$$

and

$$
\begin{aligned}
\tau^{*}(f)(y) & =\sup _{k \in \mathbf{Z}_{-}}\left[\left|\tau_{k}\right| * f(y)\right] \\
& \leq \sup _{k \in \mathbf{Z}_{-}}\left[\left(\mu_{k, \Gamma} * f\right)(y)+\left|\nu_{k}\right| * f(y)\right] \\
& \leq g_{\Gamma_{0}}(f)(y)+2 \nu^{*}(f)(y) .
\end{aligned}
$$

Following (2.22), one can apply Plancherel's theorem to yield

$$
\left\|g_{\Gamma_{0}}(f)\right\|_{2} \leq C\|f\|_{2}
$$

and hence

$$
\left\|\tau^{*}(f)\right\|_{2} \leq C\|f\|_{2}
$$

Applying the lemma on page 544 of [6] $\left(q=2, p_{0}=4\right)$, we obtain

$$
\left\|g_{\Gamma_{0}}(f)\right\|_{p} \leq C_{p}\|f\|_{p} \quad \text { for } p \in\left(\frac{4}{3}, 4\right) .
$$

By (2.19) and (2.28) we get

$$
\left\|\tau^{*}(f)\right\|_{p} \leq C_{p}\|f\|_{p} \quad \text { for } p \in\left(\frac{4}{3}, 4\right) .
$$

Repeating the process used for (2.28) with $q=\frac{4}{3}, p_{0}=8$, we get

$$
\left\|g_{\Gamma_{0}}(f)\right\|_{p} \leq C_{p}\|f\|_{p} \quad \text { for } p \in\left(\frac{8}{7}, 8\right) .
$$


Since this argument can continue as many times as one needs, we eventually have

$$
\left\|g_{\Gamma_{0}}(f)\right\|_{p} \leq C_{p}\|f\|_{p} \quad \text { for } p \in(1, \infty) .
$$

Thus by (2.24) we conclude that

$$
\left\|\mu_{\Gamma_{0}}^{*}(f)\right\|_{p} \leq\left\|g_{\Gamma_{0}}(f)\right\|_{p}+\left\|\nu^{*}(f)\right\|_{p} \leq C_{p}^{\prime}\|f\|_{p}
$$

for $1<p<\infty$. This completes the proof of Lemma 2.1.

Remark 2.2. What makes Lemma 2.1 useful is that given $\Gamma_{0}, \Gamma_{1}$, if one can verify (2.4) and (2.5), then the $L^{p}$ estimate (2.7) for $\mu_{\Gamma_{0}}^{*}$ will be derived from the $L^{p}$ estimate $(2.6)$ for $\mu_{\Gamma_{1}}^{*}$. In this context we say that $\Gamma_{0}$ is reduced to $\Gamma_{1} . \Gamma_{1}$ can be further reduced to $\Gamma_{2}$ if one can verify (2.4) and (2.5) for $\Gamma_{1}$ and $\Gamma_{2}$ with some $a_{1}>1,0<\alpha_{1}<1$ and some linear transformation $T_{1}$. This process could be extended to any finitely many steps. This is the key which enables us to prove a crucial $L^{p}$ estimate of maximal functions defined by (2.3) by reducing a realanalytic mapping to a simpler mapping whose $L^{p}$ estimate is previously known (see Theorem 4.7). Also, later we will classify (2.4) type estimates as negative power estimates and (2.5) type estimates as positive power estimates.

\section{The OsCILlatory INTEGRAL ESTIMATES}

The following lemma is a special case of Lemma 3.2 in [15].

Lemma 3.1. Let $\Psi \in C^{\infty}(\mathbf{R}), \phi \in C_{0}^{\infty}(a, b)$. Let $\lambda \in \mathbf{R} \backslash\{0\}$ and $l, m \in \mathbf{N}$ such that $\left|\Psi^{(l)}(x)\right| \leq A$ for all $x \in[a, b]$ and $\left|\Psi^{(l+1)}(x)\right| \leq B$ for all $x \in[a-A, b+A]$. Then there exists a constant $C$ which depends only on $l, A, B$ and $\phi$ such that

$$
\left|\int_{-\infty}^{\infty} e^{i \lambda \Psi(x)} \phi(x) d x\right| \leq C|\lambda|^{-\frac{\epsilon}{l}} \int_{a-A}^{b+A}\left|\Psi^{(l)}(x)\right|^{-\epsilon\left(1+\frac{1}{l}\right)} d x
$$

holds for $\lambda \in \mathbf{R}$ and $\epsilon \in[0,1]$.

Lemma 3.2 ([11, p. 76]). Let $M$ be a separable oriented real-analytic manifold and $p$ a real-analytic map from $M$ into $\mathbf{R}^{n}$. If $S$ is a real-analytic subvariety of $M$ and $K$ is a compact subset of $M$, then there exists an integer d such that

$$
\operatorname{card}\left(K \cap S \cap p^{-1}\{y\}\right) \leq d,
$$

whenever $y \in \mathbf{R}^{n}$ and $\operatorname{dim}\left(S \cap p^{-1}\{y\}\right) \leq 0$.

Lemma 3.3. For $t \in \mathbf{R}, x \in \mathbf{R}^{m}$, let $f(t, x)$ be real-analytic for $1 \leq t \leq 2$ and $|x| \leq \delta$. For $L \in \mathbf{N}$, if $\sum_{j=1}^{L}\left|\frac{\partial^{j} f}{\partial t^{j}}(t, x)\right| \geq A(x) \geq 0$ for all $1 \leq t \leq 2$ and $|x| \leq \delta$, then

$$
\left|\int_{1}^{2} e^{i f(t, x)} d t\right| \leq C(A(x))^{-\frac{1}{L}}
$$

where $C$ is independent of $x$.

Proof. For a given $x$, if $A(x)=0$, the lemma holds trivially; so assume $A(x)>0$. For positive integers $j, l$ with $1 \leq j<l \leq L$, let $F_{j l}(t, x)=\frac{\partial^{j}}{\partial t^{j}} f(t, x)-\frac{\partial^{l}}{\partial t^{t}} f(t, x)$. Let $\Sigma_{j l}=\left\{x \mid F_{j l}(t, x) \neq 0\right.$ for some $\left.t \in[1,2]\right\}$. Applying Lemma 3.2 with 
$M=\mathbf{R}^{m+1}, S=\left\{(t, x) \mid F_{j l}(t, x)=0\right\}, p:(t, x) \rightarrow x$, we see that there exists $d_{j l} \in \mathbf{N}$ such that

$$
\max _{x \in \Sigma_{j l}} \operatorname{card}\left(\left\{t \mid F_{j l, x}(t)=0, \quad t \in[1,2]\right\}\right) \leq d_{j l} .
$$

Let $D=\sum_{j, l=1}^{L} d_{j l}$. Then a standard argument yields that for all $x$ with $A(x)>0$, one can decompose the interval $[1,2]$ into at most $D$ subintervals such that on each subinterval, $\left|\frac{\partial^{j}}{\partial t^{j}} f(t, x)\right| \geq \frac{1}{L} A(x)$ for some $j, 1 \leq j \leq L$. The conclusion of the lemma follows from van der Corput's lemma (except for $j=1$, when one uses integration by parts directly) and the trivial estimate $\left|\int_{a}^{b} e^{i f(t, x)} d t\right| \leq 1$ for any $1 \leq a<b \leq 2$.

This finishes the proof of the lemma.

We need some notation. Let $B_{m}^{1}=\left\{y \in \mathbf{R}^{m} ;|y|<1\right\}$. Let $t \in \mathbf{R}, x=$ $\left(x_{1}, x^{\prime \prime}\right) \in \mathbf{R} \times \mathbf{R}^{n-2}, w=\left(x, x_{n}\right) \in \mathbf{R}^{n-1} \times \mathbf{R}$. Let $M_{n}$ be the set of all rotations in $\mathbf{R}^{n}$. It is well-known that $M_{n}$ can be embedded as a smooth compact submanifold in $\mathbf{R}^{n^{2}}$. Let $\mathbf{Z}_{-}$be the set of negative integers.

Let $\mathbf{e}=(0,0, \cdots, 1)$ be the north pole of $\mathbf{S}^{n-1}, \Omega(\cdot) \in L^{1}\left(\mathbf{S}^{n-1}\right)$ and $b \in L^{\infty}$. Let $\mathbf{F}: \mathbf{R}^{n} \rightarrow \mathbf{R}^{s}$ be real-analytic on $B_{n}^{1}$. For $\eta \in \mathbf{R}^{s} \backslash\{0\}$ and $\tilde{r} \in M_{n}$, we define $\mathbf{F}_{\tilde{r}}$ by $\mathbf{F}_{\tilde{r}}(w)=\mathbf{F}(\tilde{r} w)$ for any $w \in R^{n}$. Furthermore, define a scalar-valued function $F_{\tilde{r}, \eta^{\prime}}$ by $F_{\tilde{r}, \eta^{\prime}}(w)=\eta^{\prime} \cdot \mathbf{F}(\tilde{r} w)$ for any $w \in R^{n}$, where $\eta^{\prime}=\frac{\eta}{|\eta|}$.

For $k \in \mathbf{Z}_{-}$, set

$$
\begin{gathered}
P_{k, F_{\tilde{r}}}(\eta)=\int_{2^{k}}^{2^{k+1}} \int_{\mathbf{S}^{n-1}} e^{i|\eta| F_{\tilde{r}, \eta^{\prime}}(t w)} \Omega(w) d \sigma(w) \frac{b(t)}{t} d t \\
Q_{k, F_{\tilde{r}}}(\eta)=\int_{1}^{2}\left|\int_{\mathbf{S}^{n-1}} e^{i|\eta| F_{\tilde{r}, \eta^{\prime}}\left(2^{k} t w\right)} \Omega(w) d \sigma(w)\right|^{2} d t .
\end{gathered}
$$

In (3.1) one can dilate the variable $t$ and apply Hölder's inequality to control $P_{k, F_{\tilde{r}}}(\eta)$ in terms of $Q_{k, F_{\tilde{r}}}(\eta)$.

Lemma 3.4. Let $P_{k, F_{\tilde{r}}}(\eta)$ and $Q_{k, F_{\tilde{r}}}(\eta)$ be as in (3.1) and (3.2). Then

$$
\left|P_{k, F_{\tilde{r}}}(\eta)\right| \leq C\left[Q_{k, F_{\tilde{r}}}(\eta)\right]^{\frac{1}{2}} .
$$

Lemma 3.5. Let $\Omega \in L^{\infty}\left(\mathbf{S}^{n-1}\right)$ with $\operatorname{supp}(\Omega) \subset\left\{w \in S^{n-1} ;|w-\mathbf{e}|<\rho\right\}$ and $\|\Omega\|_{\infty} \leq \rho^{-n+1}$. For $\eta \in R^{s} \backslash\{0\}$ and $\tilde{r} \in M_{n}$, let

$$
g\left(t, x, \eta^{\prime}, \tilde{r}\right)=F_{\tilde{r}, \eta^{\prime}}\left(t x, t \sqrt{1-|x|^{2}}\right) .
$$

Assume that for $\eta_{0} \in R^{s} \backslash\{0\}$ and $\tilde{r}_{0} \in M_{n}$, there exist $l \geq 1, m \geq 1$ and $A_{0} \neq 0$ such that

$$
\begin{gathered}
\frac{\partial^{j}}{\partial x_{1}^{j}} \frac{\partial^{l} g}{\partial t^{l}}\left(0,0, \eta_{0}^{\prime}, \tilde{r}_{0}\right)=0, \quad \text { for } 0 \leq j \leq m-1 ; \\
\frac{\partial^{m}}{\partial x_{1}^{m}} \frac{\partial^{l} g}{\partial t^{l}}\left(0,0, \eta_{0}^{\prime}, \tilde{r}_{0}\right)=A_{0} .
\end{gathered}
$$

Then one can find $N_{0}>0,0<R_{0} \leq \frac{1}{2}, U_{\eta_{0}^{\prime}}$, and $V_{\tilde{r}_{0}}$ such that

$$
Q_{k, F_{\tilde{r}}}(\eta) \leq C_{0}\left(|\eta| 2^{k l(l+1)} \rho^{m(l+1)}\right)^{-\frac{1}{4 l m}}
$$


if $k \in \mathbf{Z}_{-},|k|>N_{0}, \quad 0<\rho \leq R_{0}, \eta^{\prime} \in U_{\eta_{0}^{\prime}}$, and $\tilde{r} \in V_{\tilde{r}_{0}}$, where $U_{\eta_{0}^{\prime}}$ and $V_{\tilde{r}_{0}}$ are small neighborhoods of $\eta_{0}^{\prime}$ and $\tilde{r}_{0}$ in $\mathbf{S}^{s-1}$ and $M_{n}$ respectively. Also, the constants $C_{0}, N_{0}, R_{0}$ may depend on $\eta_{0}^{\prime}, \tilde{r}_{0}$ (and hence on $l, m$, and $A_{0}$ ), but are independent of $k$ and $\rho$.

Proof. Let $f(x)=\Omega\left(x, \sqrt{1-|x|^{2}}\right)\left(1-|x|^{2}\right)^{-\frac{1}{2}}$ and choose $\phi \in C_{0}^{\infty}(0,3)$ such that $\phi(t) \geq 0$ for all $t \in \mathbf{R}$ and $\phi(t)=1$ for $1 \leq t \leq 2$. We have

$$
\begin{aligned}
Q_{k, F_{\tilde{r}}}(\eta) & \leq \int_{1}^{2}\left|\int_{B_{\rho}^{n-1}} e^{i|\eta| g\left(2^{k} t, x, \eta^{\prime}, \tilde{r}\right)} f(x) d x\right|^{2} \phi(t) d t \\
& \leq \int_{B_{\rho}^{n-1}} \int_{B_{\rho}^{n-1}}\left[\int_{-\infty}^{\infty} e^{i|\eta|\left[g\left(2^{k} t, x, \eta^{\prime}, \tilde{r}\right)-g\left(2^{k} t, y, \eta^{\prime}, \tilde{r}\right)\right]} \phi(t) d t\right] f(x) \overline{f(y)} d x d y .
\end{aligned}
$$

Denote $I_{k}\left(t, x_{1}, x^{\prime \prime}, y, \eta^{\prime}, \tilde{r}\right)=2^{-k l}\left[g\left(2^{k} t, x, \eta^{\prime}, \tilde{r}\right)-g\left(2^{k} t, y, \eta^{\prime}, \tilde{r}\right)\right]$. Then (3.3) and (3.4) give

$$
\begin{aligned}
& \frac{\partial^{j}}{\partial x_{1}^{j}} \frac{\partial^{l} I_{k}}{\partial t^{l}}\left(0,0,0,0, \eta^{\prime}, \tilde{r}\right)=0, \quad \text { for } \quad 0 \leq j \leq m-1, \\
& \frac{\partial^{m}}{\partial x_{1}^{m}} \frac{\partial^{l} I_{k}}{\partial t^{l}}\left(0,0,0,0, \eta^{\prime}, \tilde{r}\right)=A_{0} .
\end{aligned}
$$

Invoking the Malgrange Preparation Theorem $([12])$, we see that there exist $N_{0}>0,0<R_{0} \leq \frac{1}{2}, U_{\eta_{0}^{\prime}}$, and $V_{\tilde{r}_{0}}$ such that

$$
\frac{\partial^{l} I_{k}}{\partial t^{l}}\left(t, x_{1}, x^{\prime \prime}, y, \eta^{\prime}, \tilde{r}\right)=C\left(2^{k} t, x^{\prime \prime}, y, \eta^{\prime}, \tilde{r}\right)\left[x_{1}^{m}+\sum_{j=1}^{m-1} b_{j}\left(2^{k} t, x^{\prime \prime}, y, \eta^{\prime}, \tilde{r}\right) x_{1}^{j}\right]
$$

for $k \in \mathbf{Z}_{-},|k|>N_{0},\left|x_{1}\right| \leq \rho \leq R_{0},\left|x^{\prime \prime}\right| \leq \rho \leq R_{0},|y| \leq \rho \leq R_{0}, \eta^{\prime} \in U_{\eta_{0}^{\prime}}$, and $\tilde{r} \in V_{\tilde{r}_{0}}$, where $\left|C\left(2^{k} t, x^{\prime \prime}, y, \eta^{\prime}, \tilde{r}\right)\right| \geq \frac{1}{2}\left|A_{0}\right|$.

Applying Lemma 3.1 for $\epsilon=\frac{1}{4 m}$, we have

$$
\begin{aligned}
& Q_{k, F_{\tilde{r}}}(\eta) \\
& \leq C\left(2^{k l}|\eta|\right)^{-\frac{1}{4 l m}} \int_{B_{\rho}^{n-1}} \int_{B_{\rho}^{n-1}} \int_{-A}^{3+A}\left[\left|x_{1}^{m}+\sum_{j=1}^{m-1} b_{j}\left(2^{k} t, x^{\prime \prime}, y, \eta^{\prime}, \tilde{r}\right) x_{1}^{j}\right|\right]^{-\frac{l+1}{4 l m}} d t \\
& \cdot|f(x) f(y)| d x d y \\
& \leq C\left(2^{k l}|\eta|\right)^{-\frac{1}{4 l m}} \int_{-A}^{3+A} \int_{B_{\rho}^{n-1}} \int_{\left|x^{\prime \prime}\right| \leq \rho} \int_{\left|x_{1}\right| \leq \rho} \\
&\left|x_{1}^{m}+\sum_{j=1}^{m-1} b_{j}\left(2^{k} t, x^{\prime \prime}, y, \eta^{\prime}, \tilde{r}\right) x_{1}^{j}\right|^{-\frac{l+1}{4 l m}} \cdot\left|f\left(x_{1}, x^{\prime \prime}\right) f(y)\right| d x_{1} d x^{\prime \prime} d y d t \\
& \leq C\left(2^{k l}|\eta|\right)^{-\frac{1}{4 l m}} \int_{-A}^{3+A} \int_{B_{\rho}^{n-1}} \int_{\left|x^{\prime \prime}\right| \leq \rho} \int_{\left|x_{1}\right| \leq 1} \\
&\left|\left(\rho x_{1}\right)^{m}+\sum_{j=1}^{m-1} b_{j}\left(2^{k} t, x^{\prime \prime}, y, \eta^{\prime}, \tilde{r}\right)\left(\rho x_{1}\right)^{j}\right|^{-\frac{l+1}{4 l m}} \cdot \rho^{-n+1} f(y) \mid \rho d x_{1} d x^{\prime \prime} d y d t \\
& \leq C\left(2^{k l}|\eta| \rho^{m(l+1)}\right)^{-\frac{1}{4 l m}}
\end{aligned}
$$

where $C$ is independent of $k, \rho$. 
Here we used the assumption $\|\Omega\|_{\infty} \leq \rho^{-n+1}$ and the facts that $\frac{l+1}{4 l}<1$ and the constants $A$ and $B$ in Lemma 2.2 could be chosen to be independent of $k$ and $\rho$ since $k \in \mathbf{Z}_{-}$and $\rho \leq \frac{1}{2}$. This ends the proof of Lemma 3.5.

Theorem 3.6. Let $g\left(t, x, \eta^{\prime}, \tilde{r}\right)$ be defined as in Lemma 3.5. If for each $\eta^{\prime} \in$ $\mathbf{S}^{n-1}, \tilde{r} \in M_{n}$, there exist $l, m, A$ (may depend on $\eta^{\prime}$ and $\tilde{r}$ ) such that (3.3) and (3.4) hold, then one can find $L, M \geq 1, N>0,0<R \leq \frac{1}{2}$ such that

$$
\left|P_{k, F_{\tilde{r}}}(\eta)\right| \leq C\left(|\eta| 2^{k L} \rho^{M(L+1)}\right)^{-\frac{1}{8 L M}}
$$

if $k \in \mathbf{Z}_{-},|k|>N, \quad 0<\rho \leq R$, where $C$ is independent of $k, \rho, \eta$ and $\tilde{r}$.

Proof. From Lemma 3.4, it suffices to show

$$
Q_{k, F_{\tilde{r}}}(\eta) \leq C\left(|\eta| 2^{k L} \rho^{M(L+1)}\right)^{-\frac{1}{4 L M}} .
$$

Let $i, j \in \mathbf{N}$. For any $\eta_{i} \in \mathbf{R}^{s} \backslash\{0\}$ and $\tilde{r}_{j} \in M_{n}$, Lemma 3.5 asserts that there exist $l_{i, j} \geq 1, m_{i, j} \geq 1, N_{i, j}>0,0<R_{i, j} \leq \frac{1}{2}, U_{\left(\eta_{i}\right)^{\prime}}$ and $V_{\tilde{r}_{j}}$ such that

$$
Q_{k, F_{\tilde{r}}}(\eta) \leq C_{i, j}\left(|\eta| 2^{k l_{i, j}} \rho^{m_{i, j}\left(l_{i, j}+1\right)}\right)^{-\frac{1}{4 l_{i, j} m_{i, j}}}
$$

if $k \in \mathbf{Z}_{-},|k|>N_{i, j}, \quad 0<\rho \leq R_{i, j}, \quad(\eta)^{\prime} \in U_{\left(\eta_{i}\right)^{\prime}}$ and $\tilde{r} \in V_{\tilde{r}_{j}}$.

One then finds $m \geq 1$ such that $\bigcup_{i=1}^{m} U_{\left(\eta_{i}\right)^{\prime}}=\mathbf{S}^{s-1}$ and $\bigcup_{j=1}^{m} V_{\tilde{r}_{j}}=M_{n}$. Using the trivial estimate $Q_{k, F_{\tilde{r}}}(\eta) \leq C$, we obtain (3.6) by taking $L=\max _{1 \leq i, j \leq m}\left\{l_{i, j}\right\}$, $M=\max _{1 \leq i, j \leq m}\left\{m_{i, j}\right\}, N=\max _{1 \leq i, j \leq m}\left\{N_{i, j}\right\}, R=\min _{1 \leq i, j \leq m}\left\{R_{i, j}\right\}$, $C=\max _{1 \leq i, j \leq m}\left\{C_{i, j}\right\}$.

The proof of Theorem 3.6 is complete.

For real $x$ and $t$, let $f(t, x)=\sum_{j=1}^{\infty} t^{j}\left[\sum_{n=0}^{\infty} a_{j, n} x^{n}\right]$, where $a_{j, n}$ are real numbers satisfying

$$
\sum_{j=2}^{\infty}\left(\frac{1}{2}\right)^{j}\left[\sum_{n=0}^{\infty}\left|a_{j, n}\right|\left(\frac{1}{2}\right)^{n}\right]<\infty .
$$

For $|y| \leq \frac{1}{4},|x| \leq \frac{1}{4}$, we rewrite $f(t, x)$ as $\sum_{j=1}^{\infty} t^{j}\left[\sum_{m=0}^{\infty} b_{j, m}(y)(x-y)^{m}\right]$, where

$$
b_{j, m}(y)=\frac{1}{m !} \sum_{n=m}^{\infty} n(n-1) \cdots(n-m+1) a_{j, n} y^{n-m} .
$$

Since $\frac{d^{l}}{d y^{l}}\left(b_{j, m}(y)\right)=(m+l)(m+l-1) \cdots(m+1) b_{j, m+l}(y)$ and

$$
\begin{aligned}
& \sum_{m=0}^{\infty} \frac{1}{m !}\left[\sum_{n=m}^{\infty} n(n-1) \cdots(n-m+1)\left|a_{j, n}\right|\left(\frac{1}{4}\right)^{n-m}\right]\left(\frac{1}{4}\right)^{m} \\
= & \sum_{n=0}^{\infty}\left[\sum_{m=0}^{n} \frac{1}{m !} n(n-1) \cdots(n-m+1)\left(\frac{1}{2}\right)^{n}\right]\left|a_{j, n}\right|\left(\frac{1}{2}\right)^{n} \\
= & \sum_{n=0}^{\infty}\left|a_{j, n}\right|\left(\frac{1}{2}\right)^{n},
\end{aligned}
$$

it follows from (3.7) and (3.8) that if $|y| \leq \frac{1}{16},|x| \leq \frac{1}{16}$, then for each nonnegative integer $l$ there exists a constant $C_{l}$ independent of $x$ and $y$ such that

$$
\sum_{j=2}^{\infty}\left(\frac{1}{2}\right)^{j}\left[\sum_{m=0}^{\infty}\left|\frac{d^{l}}{d y^{l}}\left(b_{j, m}(y)\right) \| x-y\right|^{m}\right] \leq C_{l} .
$$


For $\bar{L} \geq 2, \bar{M} \geq 1$, define

$$
\psi(t, x, y)=\sum_{j=1}^{\bar{L}-1} t^{j}\left[\sum_{m=0}^{\infty} b_{j, m}(y)(x-y)^{m}\right]+\sum_{j=\bar{L}}^{\infty} t^{j}\left[\sum_{m=0}^{\bar{M}} b_{j, m}(y)(x-y)^{m}\right] .
$$

Let $\eta(y)$ be a continuous function of $y$. For $k \in \mathbf{Z}_{-}, \alpha(\cdot) \in L^{1}(\mathbf{R})$ and $b(\cdot) \in L^{\infty}$, set

$$
\begin{gathered}
I_{k}(y)=\int_{2^{k}}^{2^{k+1}} \int_{-\frac{1}{16}}^{\frac{1}{16}} e^{i \eta(y) \psi(t, x, y)} \alpha(x) d x \frac{b(t)}{t} d t, \\
Q_{k}(y)=\int_{1}^{2}\left|\int_{-\frac{1}{16}}^{\frac{1}{16}} e^{i \eta(y) \psi\left(2^{k} t, x, y\right)} \alpha(x) d x\right|^{2} d t .
\end{gathered}
$$

Theorem 3.7. Given $y \in\left[-\frac{1}{16}, \frac{1}{16}\right]$ and $0<\rho \leq \frac{1}{16}$, let $\alpha(\cdot) \in L^{\infty}(\mathbf{R})$ be such that $\operatorname{supp}(\alpha) \subset\{x \in \mathbf{R} ;|x-y|<\rho\}$ and $\|\alpha\|_{\infty} \leq \rho^{-1}$. If $\bar{L}>\bar{M}$ and

$$
\sum_{j=\bar{L}}^{\infty} \sum_{m=0}^{\bar{M}}\left|b_{j, m}(y)\right|>0
$$

for each $y \in\left[-\frac{1}{16}, \frac{1}{16}\right]$, then one can find $L^{\prime} \geq \bar{L}, N>0$ such that

$$
\left|I_{k}(y)\right| \leq C\left(|\eta(y)| 2^{k L^{\prime}} \rho^{\bar{M}}\right)^{-\frac{1}{2 L^{\prime}}}
$$

if $k \in \mathbf{Z}_{-},|k|>N$, where $C$ is independent of $k, \eta, y$ and $\rho$.

Proof. As in the proof of Theorem 3.6, (3.12) will be proved if one can prove

$$
\left|Q_{k}(y)\right| \leq C\left(|\eta(y)| 2^{k L^{\prime}} \rho^{\bar{M}}\right)^{-\frac{1}{L^{\prime}}} .
$$

We note that since both $\eta(y)$ and $\mathbf{Q}_{k}(y)$ are continuous, one only needs to prove (3.13) with finitely many points removed from the interval $\left[-\frac{1}{16}, \frac{1}{16}\right]$, as long as this finite number is independent of $k, \eta$, and $\rho$. For $j \geq \bar{L}$, let $\mathbf{v}_{j}(y)=$ $\left(b_{j, 0}(y), b_{j, 1}(y), \cdots, b_{j, \bar{M}}(y)\right)$ and $S(y)$ be the linear space spanned by all $\mathbf{v}_{j}(y), j \geq$ $\bar{L}$. For each $y \in\left[-\frac{1}{16}, \frac{1}{16}\right]$, let $d(y)$ be the dimension of $S(y)$ and $d=\max _{|y| \leq \frac{1}{16}} d(y)$. Since $1 \leq d(y) \leq \bar{M}+1$ for each $y$, one sees that $1 \leq d \leq \bar{M}+1$. We pick a $y_{0} \in\left[-\frac{1}{16}, \frac{1}{16}\right]$ with $d=d\left(y_{0}\right)$. For any $\left\{j_{1}, j_{2}, \cdots, j_{d}\right\}$ with $\bar{L} \leq j_{1}<$ $j_{2}<\cdots<j_{d}<\infty$, let $J=\left\{j_{1}, j_{2}, \cdots, j_{d}\right\}$. For any $y \in\left[-\frac{1}{16}, \frac{1}{16}\right]$, we form an $(\bar{M}+1) \times d$ matrix $M_{J}(y)$ by choosing the $i$-th column of $M_{J}(y)$ to be $\mathbf{v}_{j_{i}}(y)$. If $\left\{\mathbf{v}_{j_{1}}(y), \mathbf{v}_{j_{2}}(y), \cdots, \mathbf{v}_{j_{d}}(y)\right\}$ is a basis for $S(y)$, then one can find an invertible $d(y) \times d(y)$ submatrix of $M_{J}(y)$. To simplify the notation, we assume that $\left\{\mathbf{v}_{\bar{L}}\left(y_{0}\right), \mathbf{v}_{\bar{L}+1}\left(y_{0}\right), \cdots, \mathbf{v}_{\bar{L}+d-1}\left(y_{0}\right)\right\}$ is a basis of $S\left(y_{0}\right)$. We write $J_{0}=\{\bar{L}, \bar{L}+1, \cdots, \bar{L}+d-1\}$. We may also assume that the first $d$ rows of $M_{J_{0}}\left(y_{0}\right)$ give an invertible $d \times d$ submatrix at $y_{0}$. For a given $y$ and $J$, let $A_{J}(y)$ be the submatrix of $M_{J}(y)$ whose entries are the first $d$ rows of $M_{J}(y)$. Since $\operatorname{det}\left(A_{J_{0}}(y)\right)$ is real-analytic for $|y| \leq \frac{1}{16}$ and is not identically zero since $\operatorname{det}\left(A_{J_{0}}\left(y_{0}\right)\right) \neq 0$, we see that $\operatorname{det}\left(A_{J_{0}}(\cdot)\right)$ has only finitely many zeros in $\left[\frac{1}{16}, \frac{1}{16}\right]$. We cut $\left[\frac{1}{16}, \frac{1}{16}\right]$ into several subintervals, each of which contains only one zero of $\operatorname{det}\left(A_{J_{0}}(\cdot)\right)$. Without loss of generality, we may assume that the only possible zero of $\operatorname{det}\left(A_{J_{0}}(y)\right)$ is $y=0$. For 
$j>\bar{L}+d-1$ and $y \neq 0$, let $A_{J_{0}}^{j, i}(y)$ be the matrix obtained by replacing the $i$-th column of $A_{J_{0}}(y)$ by the first $d$ components of $\mathbf{v}_{j}(y)$. We are able to write

$$
\mathbf{v}_{j}(y)=\sum_{i=1}^{d} k_{j, i}(y) \mathbf{v}_{\bar{L}+i-1}(y)
$$

where $k_{j, i}(y)=\frac{\operatorname{det}\left(A_{J_{0}}^{j, i}(y)\right)}{\operatorname{det}\left(A_{J_{0}}(y)\right)}$.

For each $J$, we write

$$
\operatorname{det}\left(A_{J}(y)\right)=\sum_{u=0}^{\infty} \beta_{J, u} y^{u}
$$

Based on the property of $\operatorname{det}\left(A_{J_{0}}(y)\right)$ one can find some $\bar{J}$ and some nonnegative integer $s$ such that $\beta_{\bar{J}, s} \neq 0$, but $\beta_{J, u}=0$, for all $J$ and $0 \leq u \leq s-1$. Let the column vectors of $M_{\bar{J}}(y)$ be $\left\{\mathbf{v}_{\bar{j}_{1}}(y), \mathbf{v}_{\bar{j}_{2}}(y), \cdots, \mathbf{v}_{\bar{j}_{d}}(y)\right\}$ with $\bar{j}_{d} \geq \bar{L}+d-1$.

From the choice of $\bar{J}$ and (3.10), we see that there exists $C>0, \epsilon>0$ such that for all $|y| \leq \epsilon$ and all $j>\bar{j}_{d}$, we have

$$
\begin{gathered}
\left|\operatorname{det}\left(A_{\bar{J}}(y)\right)\right| \geq C|y|^{s}, \\
\left(\frac{1}{2}\right)^{j}\left|\operatorname{det}\left(A_{\bar{J}}^{j, i}(y)\right)\right| \leq C|y|^{s},
\end{gathered}
$$

where $\mathrm{C}$ is independent of $y$ and $j$.

Hence for $j>\bar{j}_{d}$ and $|y| \leq \epsilon$, we have

$$
\mathbf{v}_{j}(y)=\sum_{i=1}^{d} k_{j, i}(y) \mathbf{v}_{\bar{j}_{i}}(y)
$$

where $k_{j, i}(y)=\frac{\operatorname{det}\left(A_{\bar{J}}^{j, i}(y)\right)}{\operatorname{det}\left(A_{\bar{J}}(y)\right)}$.

It follows from (3.15), (3.16) and (3.17) that for $j>\bar{j}_{d}, u, v \in\left[-\frac{1}{16}, \frac{1}{16}\right]$ and $|y| \leq \epsilon$,

$$
\left(\frac{1}{2}\right)^{j}\left|\sum_{m=0}^{\bar{M}} b_{j, m}(y)\left(u^{m}-v^{m}\right)\right| \leq C \sum_{i=1}^{d}\left|\sum_{m=0}^{\bar{M}} b_{\bar{j}_{i}, m}(y)\left(u^{m}-v^{m}\right)\right|,
$$

where $C$ is independent of $u, v, y$ and $j$.

For $u, v \in\left[-\frac{1}{16}, \frac{1}{16}\right]$ and $|y| \geq \epsilon,(3.9)$ and (3.14) imply that for all $j>\bar{L}+d-1$,

$$
\left(\frac{1}{2}\right)^{j}\left|\sum_{m=0}^{\bar{M}} b_{j, m}(y)\left(u^{m}-v^{m}\right)\right| \leq C \sum_{l=\bar{L}}^{\bar{L}+d-1}\left|\sum_{m=0}^{\bar{M}} b_{l, m}(y)\left(u^{m}-v^{m}\right)\right|,
$$

where $C$ is independent of $u, v, y$ and $j$.

Combining (3.18) and (3.19) together, we see that for $u, v \in\left[-\frac{1}{16}, \frac{1}{16}\right],|y| \leq \frac{1}{16}$ and all $j>\bar{j}_{d}$,

$$
\left(\frac{1}{2}\right)^{j}\left|\sum_{m=0}^{\bar{M}} b_{j, m}(y)\left(u^{m}-v^{m}\right)\right| \leq C \sum_{l=1}^{\bar{j}_{d}}\left|\sum_{m=0}^{\bar{M}} b_{l, m}(y)\left(u^{m}-v^{m}\right)\right|
$$

holds, where $C$ is independent of $u, v, y$, and $j$. 
Let $\alpha_{y}(u)=\alpha(u+y)$ and

$$
\psi_{y}(t, u)=\sum_{j=1}^{\bar{L}-1} t^{j}\left[\sum_{m=0}^{\infty} b_{j, m}(y) u^{m}\right]+\sum_{j=\bar{L}}^{\infty} t^{j}\left[\sum_{m=0}^{\bar{M}} b_{j, m}(y) u^{m}\right] .
$$

One implication of (3.20) is that there exist $C>0, N>0$ such that for $k \in$ $\mathbf{Z}_{-},|k|>N, t \in[1,2], u, v \in\left[-\frac{1}{16}, \frac{1}{16}\right]$ and $|y| \leq \frac{1}{16}$, we have

$$
\begin{aligned}
\sum_{l=\bar{L}}^{\bar{j}_{d}} \mid & \frac{\partial^{(l)}}{\partial t^{(l)}} \psi_{y}\left(2^{k} t, u\right)-\psi_{y}\left(2^{k} t, v\right) \mid \\
& \geq C 2^{-k \bar{j}_{d}} \sum_{l=\bar{L}}^{\bar{j}_{d}}\left|\sum_{m=0}^{\bar{M}} b_{l, m}(y)\left(u^{m}-v^{m}\right)\right|,
\end{aligned}
$$

where $C$ is independent of $k, t, u, v$ and $y$.

We rewrite $Q_{k}(y)$ as

$$
Q_{k}(y)=\int_{-\rho}^{\rho} \int_{-\rho}^{\rho} \int_{1}^{2} e^{i \eta(y)\left[\psi_{y}\left(2^{k} t, u\right)-\psi\left(2^{k} t, v\right)\right]} d t \alpha_{y}(u) \overline{\alpha_{y}(v)} d u d v .
$$

Then (3.21), the assumptions on $\alpha(\cdot)$, Lemma 3.3 and a result of Ricci and Stein in 18 yield

$$
\begin{aligned}
\left|Q_{k}(y)\right| \leq & C\left(|\eta(y)| 2^{k \bar{j}_{d}}\right)^{-\frac{1}{j_{d}}} \int_{-\rho}^{\rho} \int_{-\rho}^{\rho}\left[\sum_{l=\bar{L}}^{\bar{j}_{d}} \mid \sum_{m=0}^{\bar{M}} b_{l, m}(y) u^{m}\right. \\
& \left.-\sum_{m=0}^{\bar{M}} b_{l, m}(y) v^{m} \mid\right]^{-\frac{1}{j_{d}}} \rho^{-2} d u d v \\
\leq & C\left(|\eta(y)| 2^{k \bar{j}_{d}}\right)^{-\frac{1}{j_{d}}} \int_{-1}^{1}\left[\int _ { - 1 } ^ { 1 } \left[\sum_{l=\bar{L}} \mid \sum_{m=0}^{\bar{M}} b_{l, m}(y) \rho^{m} u^{m}\right.\right. \\
& \left.\left.-\sum_{m=0}^{\bar{M}} b_{l, m}(y) \rho^{m} v^{m} \mid\right]^{-\frac{1}{j_{d}}} d u\right] d v \\
\leq & C\left(|\eta(y)| 2^{k \bar{j}_{d}}\right)^{-\frac{1}{\bar{j}_{d}}}\left[\sum_{l=\bar{L}}^{\bar{j}_{d}} \sum_{m=0}^{\bar{M}}\left|b_{l, m}(y) \rho^{m}\right|\right]^{-\frac{1}{\bar{j}_{d}}} \\
\leq & C\left(|\eta(y)| 2^{k \bar{j}_{d}} \rho^{\bar{M}}\right)^{-\frac{1}{\bar{j}_{d}}}\left[\sum_{l=\bar{L}}^{\overline{j_{d}}} \sum_{m=0}^{\bar{M}}\left|b_{l, m}(y)\right|\right]^{-\frac{1}{j_{d}}} \\
\leq & C\left(|\eta(y)| 2^{k \bar{j}_{d}} \rho^{\bar{M}}\right)^{-\frac{1}{\bar{j}_{d}}},
\end{aligned}
$$

where $C$ is independent of $k, \eta, y$ and $\rho$.

Here in the third inequality, we used the assumption that $\bar{L}>\bar{M}$ and in the last inequality, we used (3.21) and the assumption that $\sum_{j=\bar{L}}^{\infty} \sum_{m=0}^{\bar{M}}\left|b_{j, m}(y)\right|>0$ for each $y \in\left[-\frac{1}{16}, \frac{1}{16}\right]$.

The proof of Theorem 3.7 will be finished if one chooses $L^{\prime}$ to be $\bar{j}_{d}$. 


\section{The PRoOf OF MAIN RESUlts}

Definition 4.1. A function $a(\cdot)$ on $\mathbf{S}^{n-1}$ is called a regular atom if there exist $\zeta \in \mathbf{S}^{n-1}$ and $\rho \in(0,2]$ such that

$$
\begin{gathered}
\operatorname{supp}(a) \subset\left\{w \in \mathbf{S}^{n-1}:|w-\zeta|<\rho\right\} ; \\
\|a\|_{\infty} \leq \rho^{-1} ; \\
\int_{\mathbf{S}^{n-1}} a(w) d \sigma(w)=0 .
\end{gathered}
$$

Let $\mathcal{S}\left(\mathbf{S}^{n-1}\right)$ be the Schwartz space of smooth functions on $\mathbf{S}^{n-1}$ and $\mathcal{S}^{\prime}\left(\mathbf{S}^{n-1}\right)$ its dual. For $f \in \mathcal{S}^{\prime}$ and the Poisson kernel $P_{r y}(x)=\frac{1-r^{2}}{|r y-x|^{n}}$ on $\mathbf{S}^{n-1}$, we define its radial maximal function $P^{+} f$ by

$$
P^{+} f(x)=\sup _{0 \leq r<1}\left|\int_{\mathbf{S}^{n-1}} P_{r x}(y) f(y) d \sigma(y)\right|
$$

where $x \in \mathbf{S}^{n-1}$. The Hardy space $H^{1}\left(\mathbf{S}^{n-1}\right)$ is defined by

$$
H^{1}\left(\mathbf{S}^{n-1}\right)=\left\{f \in \mathcal{S}^{\prime}\left(\mathbf{S}^{n-1}\right):\left\|P^{+} f\right\|_{L^{1}\left(\mathbf{S}^{n-1}\right)}<\infty\right\}
$$

with $\|f\|_{H^{1}\left(\mathbf{S}^{n-1}\right)}=\left\|P^{+} f\right\|_{L^{1}\left(\mathbf{S}^{n-1}\right)}$. We will make use of the following property of $H^{1}\left(\mathbf{S}^{n-1}\right)$ (see [3] or [4]).

Lemma 4.2. For any $f \in H^{1}\left(\mathbf{S}^{n-1}\right)$ with $\int_{S^{n-1}} f(w) d \sigma(w)=0$, there are complex numbers $c_{j}$ and regular atoms $a_{j}$ such that

$$
f=\sum_{j} c_{j} a_{j}
$$

and $\|f\|_{H^{1}} \approx \sum_{j}\left|c_{j}\right|$.

Using (2.8) in section 2, one can easily verify the following extension of a result due to Duoandikoetxea and Rubio de Francia [6].

Lemma 4.3. Let $d$ and $m$ be two positive integers and $L: \mathbf{R}^{d} \rightarrow \mathbf{R}^{m}$ a linear transformation. Suppose $\left\{\sigma_{k}\right\}_{k \in \mathbf{Z}}$ is a sequence of measures on $\mathbf{R}^{d}$ satisfying

(i) $\left\|\sigma_{k}\right\| \leq 1$ for $k \in \mathbf{Z}$.

(ii) $\left|\hat{\sigma}_{k}(\xi)\right| \leq C\left[\min \left\{a_{k x c}|L \xi|,\left(a_{k}|L \xi|\right)^{-1}\right\}\right]^{\alpha}$ for $\xi \in \mathbf{R}^{d}$ and $k \in \mathbf{Z}$.

(iii) For some $1<q<\infty$, the operator $\sigma^{*}: f \rightarrow \sigma^{*}(f)$ is bounded from $L^{q}\left(\mathbf{R}^{d}\right)$ to itself.

Then for $p \in\left(\frac{2 q}{q+1}, \frac{2 q}{q-1}\right)$, there exists a constant $C_{p}=C(p, d, m)$ which is independent of $L$ such that

$$
\left\|\sum_{k=-\infty}^{\infty} \sigma_{k} * f\right\|_{p} \leq C_{p}\|f\|_{p}
$$

and

$$
\left\|\left(\sum_{k=-\infty}^{\infty}\left|\sigma_{k} * f\right|^{2}\right)^{\frac{1}{2}}\right\|_{p} \leq C_{p}\|f\|_{p}
$$

for every $f \in L^{p}\left(\mathbf{R}^{d}\right)$.

Let $\Gamma(t): \quad[0,1] \rightarrow \mathbf{R}$. For $f \in L^{p}\left(\mathbf{R}^{2}\right)$ for some $1 \leq p \leq \infty$, we define

$$
\left(M_{\Gamma} f\right)\left(x_{1}, x_{2}\right)=\sup _{0<h<1} \frac{1}{h} \int_{0<t<h}\left|f\left(x_{1}-t, x_{2}-\Gamma(t)\right)\right| d t .
$$


Lemma 4.4 ([24, Theorem $12(\mathrm{~B})])$. If $\Gamma$ is analytic, then

$$
\left\|M_{\Gamma} f\right\|_{p} \leq C_{p}\|f\|_{p}, \quad 1<p<\infty .
$$

For a positive integer $m$ we let $V_{m}$ denote the space of real-valued homogeneous polynomials of degree $m$ on $\mathbf{R}^{n}$ and set $N_{m}=\operatorname{dim}\left(V_{m}\right)$. For

$$
P(y)=\sum_{|\alpha|=m} a_{\alpha} y^{\alpha} \in V_{m}
$$

we define

$$
\|P\|=\sum_{|\alpha|=m}\left|a_{\alpha}\right| .
$$

If $m$ is an even, positive integer, then we have

$$
|x|^{m}=\left(x_{1}^{2}+x_{2}^{2}+\cdots+x_{n}^{2}\right)^{m / 2} \in V_{m} .
$$

We now choose a basis $\left\{\zeta_{1}, \zeta_{2}, \ldots, \zeta_{N_{m}}\right\}$ for the space $V_{m}$ such that $\zeta_{1}(x)=|x|^{m}$ for $x \in \mathbf{R}^{n}$. Clearly, there are constants $K_{1}, K_{2}$ such that

$$
K_{1} \sum_{j=1}^{N_{m}}\left|c_{j}\right| \leq\|P\| \leq K_{2} \sum_{j=1}^{N_{m}}\left|c_{j}\right|
$$

for every

$$
P=\sum_{j=1}^{N_{m}} c_{j} \zeta_{j} \in V_{m} .
$$

For the above polynomial $P$, we define the linear transformation $Y_{m}: V_{m} \rightarrow V_{m}$ by

$$
Y_{m}(P)=\sum_{j=2}^{N_{m}} c_{j} \zeta_{j}
$$

Also define the linear transformation $Z_{m}: V_{m} \rightarrow V_{m}$ by

$$
Z_{m}= \begin{cases}i d_{V_{m}} & \text { if } m \text { is odd } \\ Y_{m} & \text { if } m \text { is even. }\end{cases}
$$

Lemma 4.5 (9, Proposition 5.1]). Let $b \in L^{\infty}, \Omega \in L^{2}\left(\mathbf{S}^{n-1}\right)$. Suppose $F: \mathbf{R}^{n} \rightarrow$ $\mathbf{R}$ is a function given by

$$
\sum_{j=0}^{l} P_{j}(w)+W(|w|),
$$

where $P_{j}(\cdot)$ is a homogeneous polynomial of degree $j, 0 \leq j \leq l$, and $W(\cdot)$ is an arbitrary function. Then we have

$$
\begin{aligned}
\int_{2^{k}}^{2^{k+1}} & \left|\int_{\mathbf{S}^{n-1}} e^{i F(t w)} \Omega(w) d \sigma(w)\right| \frac{|b(t)|}{t} d t \\
\leq & C\left(2^{k l}\left\|Z_{l}\left(P_{l}\right)\right\|\right)^{-\frac{1}{8 l}\|\Omega\|_{2}}
\end{aligned}
$$

for all $k \in \mathbf{Z}$. The constant $C$ is independent of $k, \Omega(\cdot), W(\cdot)$, and the coefficients of $P_{j}(\cdot)$. 
For $y=\left(y_{1}, \ldots, y_{n}\right) \in \mathbf{R}^{n}$ we let $\tilde{y}=\left(y_{1}, \ldots, y_{n-1}\right) \in \mathbf{R}^{n-1}$. We shall consider the functions $F: \mathbf{R} \times \mathbf{R}^{n} \rightarrow \mathbf{R}$ of the form

$$
F(t, y)=t^{l} q(\tilde{y})+W_{1}(t, y)+W_{2}(t)
$$

where $q: \mathbf{R}^{n-1} \rightarrow \mathbf{R}$ is a polynomial, $W_{1}$ satisfies

$$
\frac{\partial^{l} W_{1}}{\partial t^{l}}(t, y) \equiv 0
$$

and $W_{2}(\cdot)$ is an arbitrary function.

Lemma 4.6 ([9] Proposition 5.3]). Let $\rho \in(0,1 / 4), l \in \mathbf{N}, m \geq 0, q(\tilde{y})=\sum_{j=0}^{m} q_{j}(\tilde{y})$, where $q_{j}(\cdot)$ is a homogeneous polynomial of degree $j$ on $\mathbf{R}^{n-1}$ for $0 \leq j \leq m$. Let $F(t, y)$ be given by (4.3) and (4.4). Suppose that $b \in L^{\infty}$ and $\Omega(\cdot)$ is a function satisfying

$$
\operatorname{supp}(\Omega) \subset\left\{w \in \mathbf{S}^{n-1} ;|w-\mathbf{e}|<\rho\right\}
$$

and

$$
\|\Omega\|_{\infty} \leq \rho^{-n+1}
$$

If we assume $q_{m}(\tilde{y})=\sum_{|\beta|=m} a_{\beta} \tilde{y}^{\beta}$ and $\left\|q_{m}\right\|=\sum_{|\beta|=m}\left|a_{\beta}\right|$, then there exists a positive constant $C$ such that

$$
\int_{2^{k}}^{2^{k+1}}\left|\int_{\mathbf{S}^{n-1}} e^{i F(t, y)} \Omega(y) d \sigma(y)\right| \frac{|b(t)|}{t} d t \leq C\left(2^{k l} \rho^{m}\left\|q_{m}\right\|\right)^{-\frac{1}{4 m l}} .
$$

The constant $C$ may depend on $l, m, n$, and $b(\cdot)$, but it is independent of $k, \rho$, $W_{1}(\cdot, \cdot), W_{2}(\cdot)$, and the coefficients of $q(\cdot)$.

Theorem 4.7. Let $w=(x, z) \in \mathbf{R}^{2}$ and $\psi(w)$ be real-analytic on $B_{2}^{1}$ with $\psi(0)=$ $\nabla \psi(0)=0$. Let $\boldsymbol{\Psi}(w)=(w, \psi(w))$ and $\mu_{\mathbf{\Psi}}^{*}$ be as defined in (2.3). If $b \in L^{\infty}(\mathbf{R})$ and $\Omega$ is a regular atom defined in Definition 4.1 , then

$$
\left\|\mu_{\Psi}^{*} f\right\|_{p} \leq C_{p}\|f\|_{p}, \quad 1<p \leq \infty
$$

for $f \in L^{p}\left(\mathbf{R}^{d}\right)$, where $C_{p}$ is independent of $\zeta$ and $\rho$.

Proof. For $p=\infty,(4.8)$ is trivial; so we only consider $1<p<\infty$. We assume $\rho<R_{0} \leq \frac{1}{16}$, where $R_{0}$ is a small positive number to be fixed later. For $\rho \geq R_{0}$, the proof is similar (indeed easier) as one can see from the proof of Theorem 1.1 in 9]. We divide our discussion into two cases.

Case 1: $\psi$ is radial.

Since $\psi$ is rotation-invariant, after a suitable rotation in $\mathbf{R}^{2}$, we may assume that $\operatorname{supp}(\Omega) \subset\left\{w \in \mathbf{S}^{1} ;|w-\mathbf{e}|<\rho\right\}$. Let $\xi=\left(\xi_{1}, \xi_{2}, \xi_{3}\right) \in \mathbf{R}^{3}$. In Lemma 4.5, we take $l=1$ to get

$$
\left|\hat{\mu}_{k, \Psi}(\xi)\right| \leq C\left[2^{k}\left(\left|\xi_{1}\right|+\left|\xi_{2}\right|\right) \rho^{4}\right]^{-\frac{1}{8}}
$$

for $k \in \mathbf{Z}_{-}$, where we used the fact that $\|\Omega\|_{2}=C \rho^{-\frac{1}{2}}$.

For $t \in[0,1], \quad|x|<\frac{2}{3}$, we write $w$ in polar coordinates, namely, let $w=$ $\left(t x, t \sqrt{1-x^{2}}\right)$ so that locally we have $\boldsymbol{\Psi}=\left\{\left(t x, t \sqrt{1-x^{2}}, \psi\left(t x, t \sqrt{1-x^{2}}\right)\right), t \in\right.$ $\left.[0,1],|x|<\frac{2}{3}\right\}$. Since $\psi$ is radial, we have $\psi\left(t x, t \sqrt{1-x^{2}}\right)=\psi(t)$. Since the 
power series expansion of $\sqrt{1-x^{2}}$ is $1-\frac{1}{2} x^{2}-\frac{1}{8} x^{4}-\cdots$, we define $\boldsymbol{\Psi}_{1}=(t x, t(1-$ $\left.\left.\frac{1}{2} x^{2}\right), \psi(t)\right)$. It follows that

$$
\left|\hat{\mu}_{k, \Psi}(\xi)-\hat{\mu}_{k, \Psi_{1}}(\xi)\right| \leq C\left[2^{k}\left(\left|\xi_{1}\right|+\left|\xi_{2}\right|\right) \rho^{4}\right]
$$

for $k \in \mathbf{Z}_{-}$.

From Lemma 2.1, we see that (4.9) and (4.10) reduce $\boldsymbol{\Psi}$ to $\boldsymbol{\Psi}_{1}$ since one can take $a_{0}=2, T_{0} \xi=\left(\rho^{4} \xi_{1}, \rho^{4} \xi_{2}, 0\right)$. Invoking Lemma 4.6 with $l=1$ and $m=2$, we have

$$
\left|\hat{\mu}_{k, \mathbf{\Psi}_{1}}(\xi)\right| \leq C\left[2^{k}\left|\xi_{2}\right| \rho^{2}\right]^{-\frac{1}{8}}
$$

If we define $\boldsymbol{\Psi}_{2}=(t x, t, \psi(t))$, then we have

$$
\left|\hat{\mu}_{k, \boldsymbol{\Psi}_{1}}(\xi)-\hat{\mu}_{k, \boldsymbol{\Psi}_{2}}(\xi)\right| \leq C\left[2^{k}\left|\xi_{2}\right| \rho^{2}\right] .
$$

Thus Lemma 2.1 reduces $\boldsymbol{\Psi}_{1}$ to $\boldsymbol{\Psi}_{2}$. We apply Lemma 4.6 again with $l=1$ and $m=1$ to get

$$
\left|\hat{\mu}_{k, \Psi_{2}}(\xi)\right| \leq C\left[2^{k}\left|\xi_{1}\right| \rho\right]^{-\frac{1}{4}} .
$$

Then we define $\mathbf{\Psi}_{3}=(0, t, \psi(t))$ so that we have

$$
\left|\hat{\mu}_{k, \Psi_{2}}(\xi)-\hat{\mu}_{k, \Psi_{3}}(\xi)\right| \leq C\left[2^{k}\left|\xi_{1}\right| \rho\right] .
$$

It follows that we have reduced $\boldsymbol{\Psi}_{2}$ to $\boldsymbol{\Psi}_{3}$.

For fixed $x_{1}$, let $g\left(x_{2}, x_{3}\right)=f\left(x_{1}, x_{2}, x_{3}\right)$. We observe that $\mu_{\mathbf{\Psi}_{3}}^{*} f\left(x_{1}, x_{2}, x_{3}\right) \leq$ $C \int_{S^{1}} M_{\psi}(|g|)\left(x_{2}, x_{3}\right)|\Omega(y)| d \sigma(y) \leq C M_{\psi}(|g|)\left(x_{2}, x_{3}\right)\|\Omega\|_{1} \leq C M_{\psi}(|g|)\left(x_{2}, x_{3}\right)$.

Applying Lemma 4.4 for $M_{\psi}(|g|)\left(x_{2}, x_{3}\right)$ and $1<p<\infty$, we have

$$
\begin{aligned}
\left\|\mu_{\mathbf{\Psi}_{3}}^{*} f\right\|_{p}^{p} & =\int_{R^{2}} \int_{R^{1}}\left(\mu_{\mathbf{\Psi}_{3}}^{*} f\right)^{p}\left(x_{1}, x_{2}, x_{3}\right) d x_{2} d x_{3} d x_{1} \\
& \leq C \int_{R^{2}} \int_{R^{1}}\left(M_{\psi}(|g|)\left(x_{2}, x_{3}\right)\right)^{p} d x_{2} d x_{3} d x_{1} \\
& \leq C \int_{R^{2}} \int_{R^{1}}\left|g\left(x_{2}, x_{3}\right)\right|^{p} d x_{2} d x_{3} d x_{1} \\
& \leq C \int_{R^{2}} \int_{R^{1}}\left|f\left(x_{1}, x_{2}, x_{3}\right)\right|^{p} d x_{1} d x_{2} d x_{3} \\
& =C\|f\|_{p}^{p} .
\end{aligned}
$$

This finishes the proof of case 1 .

Case 2: $\psi$ is not radial.

Let $\zeta$ be as in (2.1). Without loss of generality, we may assume that $|\zeta-\mathbf{e}|<$ $\frac{1}{16}$. Let $\zeta=\left(y, \sqrt{1-y^{2}}\right)$ so that $|y|<\frac{1}{16}$. It follows that if we let $\alpha(x)=$ $\Omega\left(x, \sqrt{1-x^{2}}\right)\left(1-x^{2}\right)^{-\frac{1}{2}}$, then $\operatorname{supp}(\alpha) \subset\{x|| x-y \mid<\rho\}$ and $\|\alpha\| \leq 2 \rho^{-1}$.

Let $B_{2}^{1}$ be the unit ball of $\mathbf{R}^{2}$. Since $\psi(w)$ is real-analytic on $B_{2}^{1}$, we have

$$
\psi(w)=\sum_{j=2}^{\infty} \sum_{|\alpha|=j} c_{\alpha} w^{\alpha} .
$$

Let $f(t, x)=\psi\left(t x, t \sqrt{1-x^{2}}\right)$. It follows that

$$
f(t, x)=\sum_{j=2}^{\infty} t^{j}\left[\sum_{n=0}^{\infty} a_{j, n} x^{n}\right],
$$

where $a_{j, n}$ satisfies (3.7). 
One can rewrite $f(t, x)$ as

$$
f(t, x)=\sum_{j=2}^{\infty} t^{j}\left[\sum_{m=0}^{\infty} b_{j, m}(y)(x-y)^{m}\right],
$$

where $b_{j, m}(y)$ is defined by (3.8).

We point out that the rotation $\tilde{r}_{0}$ on $R^{2}$ in Theorem 3.6 is equivalent to the translation of the variable $x$ by $y$. Let $g\left(t, x, \xi^{\prime}, y\right)=t\left[\xi_{1}^{\prime} x+\xi_{2}^{\prime} \sqrt{1-x^{2}}\right]+\xi_{3}^{\prime} f(t, x)$. We have

$$
\hat{\mu}_{k, \Psi}(\xi)=\int_{2^{k}}^{2^{k+1}} \int_{-\rho}^{\rho} e^{i|\xi| g\left(t, x, \xi^{\prime}, y\right)} \alpha(x) d x \frac{b(t)}{t} d t .
$$

Since $\psi$ is not radial, we see that $f(t, x)$ depends on $x$. Thus for each $y$ with $|y|<\frac{1}{4}$, one can find $b_{j, m}(y) \neq 0$ for some $j \geq 2$ and some $m>0$. It follows that the function $g$ satisfies the conditions (3.3) and (3.4). Now we fix $R_{0}$ so small that Theorem 3.6 can be applied. Thus, one can find $L, M \geq 1, N>0$ and $\alpha_{0}>0$ such that

$$
\left|\hat{\mu}_{k, \Psi}(\xi)\right| \leq C\left(|\xi| 2^{k L} \rho^{M(L+1)}\right)^{-\alpha_{0}}
$$

if $k \in \mathbf{Z}^{-},|k|>N, \quad 0<\rho \leq R_{0}$.

Let

$$
f_{1}(t, x)=\sum_{j=2}^{L-1} t^{j}\left[\sum_{m=0}^{\infty} b_{j, m}(y)(x-y)^{m}\right]+\sum_{L}^{\infty} t^{j}\left[\sum_{m=0}^{M(L+1)-1} b_{j, m}(y)(x-y)^{m}\right]
$$

and define the mapping $\boldsymbol{\Psi}_{1}$ by

$$
\boldsymbol{\Psi}_{1}(t, x)=\left(t x, t \sqrt{1-x^{2}}, f_{1}(t, x)\right) .
$$

The above definition together with (4.13) yields

$$
\left|\hat{\mu}_{k, \boldsymbol{\Psi}}(\xi)-\hat{\mu}_{k, \mathbf{\Psi}_{1}}(\xi)\right| \leq C\left(|\xi| 2^{k L} \rho^{M(L+1)}\right)
$$

if $0<\rho \leq R_{0}$, where $C$ is independent of $k, \rho, \xi$ and $y$.

Having obtained (4.14) and (4.16), we have reduced $\boldsymbol{\Psi}$ to $\boldsymbol{\Psi}_{1}$ by choosing $T_{0} \xi=$ $\rho^{M(L+1)} \xi$ and $a=2^{L}$. If the second summation of $f_{1}(t, x)$ is zero, (4.11), (4.12) and (4.13) imply that $\Psi_{1}=\left(w, \sum_{j=2}^{L-1} \sum_{|\alpha|=j} c_{\alpha} w^{\alpha}\right)$, which is covered by Theorem 7.4 in [9] since in this case $\boldsymbol{\Psi}_{1}$ is a polynomial mapping. Thus, we may assume that the second summation of $f_{1}(t, x)$ is not identically zero.

To reduce $\boldsymbol{\Psi}_{1}$ further, one can employ Theorem 3.7 to get the desired negative power estimate for $\hat{\mu}_{k, \boldsymbol{\Psi}_{1}}(\xi)$. If $\sum_{L}^{\infty} \sum_{m=0}^{M(L+1)-1}\left|b_{j, m}\left(y_{0}\right)\right|=0$ for some $y_{0}$, then one can find $d \in \mathbf{N}$ such that $b_{j, m}(y)=\left(y-y_{0}\right)^{d} c_{j, m}(y)$ and $\sum_{L}^{\infty} t^{j}\left[\sum_{m=0}^{M(L+1)-1}\left|c_{j, m}(y)\right|\right]$ $>0$ in a neighborhood of $y_{0}$. Since $b_{j, m}(y)$ is analytic, by decomposing the interval of $y$ into several subintervals if necessary, we may assume that $y_{0}$ is the only common zero of $b_{j, m}(y)$. Thus by Theorem 3.7, one can find $L_{1} \geq L, N>0$ and $\alpha_{1}>0$ such that

$$
\left|\hat{\mu}_{k, \Psi_{1}}(\xi)\right| \leq C\left(\left|\xi_{3}\left(y-y_{0}\right)^{d}\right| 2^{k L_{1}} \rho^{M(L+1)-1}\right)^{-\alpha_{1}}
$$

if $k \in \mathbf{Z}_{-},|k|>N, \quad 0<\rho \leq \frac{1}{16}$. 
Let

$$
\begin{aligned}
f_{2}(t, x) & =\sum_{j=2}^{L-1} t^{j}\left[\sum_{m=0}^{\infty} b_{j, m}(y)(x-y)^{m}\right]+\sum_{j=L}^{L_{1}-1} t^{j}\left[\sum_{m=0}^{M(L+1)-1} b_{j, m}(y)(x-y)^{m}\right] \\
& +\sum_{L_{1}}^{\infty} t^{j}\left[\sum_{m=0}^{M(L+1)-2} b_{j, m}(y)(x-y)^{m}\right],
\end{aligned}
$$

where the $\sum_{j=L}^{L_{1}-1}$ term is zero if $L_{1}=L$. We define the mapping $\Psi_{2}$ by

$$
\boldsymbol{\Psi}_{2}=\left(t x, t \sqrt{1-x^{2}}, f_{2}(t, x)\right)
$$

It follows from (4.15) and (4.18) that

$$
\left|\hat{\mu}_{k, \boldsymbol{\Psi}_{1}}(\xi)-\hat{\mu}_{k, \boldsymbol{\Psi}_{2}}(\xi)\right| \leq C\left(\left|\xi_{3}\left(y-y_{0}\right)^{d}\right| 2^{k L_{1}} \rho^{M(L+1)-1}\right)
$$

if $0<\rho \leq R$, where $C$ is independent of $k, \rho, \xi$ and $y$.

Then one applies Lemma 2.1 with $T_{1} \xi=\left(y-y_{0}\right)^{d} \rho^{M(L+1)-1} \xi_{3}$ and $a_{1}=2^{L_{1}}$ to see that $\boldsymbol{\Psi}_{1}$ has been reduced to $\boldsymbol{\Psi}_{2}$. Repeating this process at most $M(L+1)$ times, one can reduce $\boldsymbol{\Psi}$ to a mapping $\boldsymbol{\Phi}$ given by

$$
\mathbf{\Phi}=\left(t x, t \sqrt{1-x^{2}}, g(t, x, y)\right)
$$

with

$$
\begin{aligned}
g(t, x, y) & =\sum_{j=2}^{L-1} t^{j}\left[\sum_{m=0}^{\infty} b_{j, m}(y)(x-y)^{m}\right]+\sum_{L}^{L_{1}-1} t^{j}\left[\sum_{m=0}^{M(L+1)-1} b_{j, m}(y)(x-y)^{m}\right] \\
& +\sum_{l=1}^{M(L+1)-1} \sum_{L_{l}}^{\left.L_{l+1}-1\right)} t^{j}\left[\sum_{m=0}^{M(L+1)-(l+1)} b_{j, m}(y)(x-y)^{m}\right]+\sum_{j=L_{M(L+1)}}^{\infty} b_{j, 0} t^{j},
\end{aligned}
$$

where $L_{1} \leq L_{2} \leq \cdots \leq L_{M(L+1)}$.

Based on Lemma 4.6 and Lemma 2.1, one then applies the argument that reduced $\boldsymbol{\Psi}_{1}$ to $\boldsymbol{\Psi}_{2}$ finitely many times to see that $\boldsymbol{\Phi}$ can be reduced to $\boldsymbol{\Phi}_{1}$, where $\boldsymbol{\Phi}_{1}$ is defined by

$$
\mathbf{\Phi}_{1}=\left(t x, t \sqrt{1-x^{2}}, g_{1}(t, x, y)\right)
$$

with

$$
g_{1}(t, x, y)=\sum_{j=2}^{L-1} t^{j}\left[\sum_{m=0}^{\infty} b_{j, m}(y)(x-y)^{m}\right]+\sum_{j=L}^{\infty} b_{j, 0}(y) t^{j} .
$$

Again from (4.11), (4.12) and (4.13), we see that $\boldsymbol{\Phi}_{1}=\left(w, \sum_{j=2}^{L-1} \sum_{|\alpha|=j} c_{\alpha} w^{\alpha}+\right.$ $\left.h_{1}(|w|)\right)$, where $h(|w|)=\sum_{j=L}^{\infty} b_{j, 0}(y)|w|^{j}$. Similar to the process of reducing $\boldsymbol{\Psi}$ to $\boldsymbol{\Psi}_{1}$ and then to $\boldsymbol{\Psi}_{2}$, we can combine Lemma 4.2, Lemma 4.3 and Lemma 2.2 to reduce $\boldsymbol{\Phi}_{1}$ to $\boldsymbol{\Phi}_{2}$ and then to $\boldsymbol{\Phi}_{3}$, where

$$
\mathbf{\Phi}_{2}(w)=\left(w, h_{2}(|w|)\right)
$$

with $h_{2}(|w|)=\sum_{j=2}^{\infty} b_{j, 0}(y)|w|^{j}$ and

$$
\mathbf{\Phi}_{3}(w)=\left(0,|w|, h_{2}(|w|)\right)=\left(0, t, h_{2}(t)\right) .
$$

We point out that one cannot apply Lemma 4.4 directly to $\boldsymbol{\Phi}_{3}$ since the coefficients of $h_{2}(t)$ are dependent on $y$ and our desired estimate (4.8) should be 
independent of $y$. Thus we need to apply Lemma 2.1 to reduce $\boldsymbol{\Phi}_{3}$ further. From the definition of $\mu_{\mathbf{\Phi}_{3}}^{*}$, we see that, without loss of generality, we may assume that $b(t) \equiv 1$. It follows that

$$
\hat{\mu}_{k, \mathbf{\Phi}_{3}}(\xi)=\int_{\mathbf{S}^{1}}\left[\int_{2^{k}}^{2^{k+1}} e^{i\left(\xi_{2} t+\xi_{3} h_{2}(t)\right)} \frac{1}{t} d t\right]|\Omega(w)| d \sigma(w) .
$$

Also, if necessary we can pull out a factor $\left(y-y_{0}\right)$ from $b_{j, 0}(y)$ so that we may assume $\sum_{j=2}^{\infty}\left|b_{j, 0}(y)\right|>0$ for all $|y| \leq \frac{1}{16}$. This combined with van der Corput's lemma yields that there exist $J, J_{1}, N \in \mathbf{N} \backslash\{1\}$ such that

$$
\begin{aligned}
\left|\int_{2^{k}}^{2^{k+1}} e^{i\left(\xi_{2} t+\xi_{3} h_{2}(t)\right)} \frac{1}{t} d t\right| & =\left|\int_{1}^{2} e^{i\left(\xi_{2} 2^{k} t+\xi_{3} h_{2}\left(2^{k} t\right)\right)} \frac{1}{t} d t\right| \\
& \leq C\left(\left|\xi_{3}\right| 2^{J k}\right)^{-\frac{1}{J_{1}}}
\end{aligned}
$$

for all $k \in \mathbf{Z}_{-},|k|>N$.

Since $\Omega \in L^{1}\left(\mathbf{S}^{1}\right)$, (4.24) yields

$$
\left|\hat{\mu}_{k, \Phi_{3}}(\xi)\right| \leq C\left(\left|\xi_{3}\right| 2^{J k}\right)^{-\frac{1}{J_{1}}}
$$

for all $k \in \mathbf{Z}_{-},|k|>N$, where $C$ is independent of $k, \xi, \rho$ and $y$.

We define $\boldsymbol{\Phi}_{4}$ by

$$
\boldsymbol{\Phi}_{4}(w)=\left(0,|w|, \sum_{j=2}^{J} b_{j, 0}(y)|w|^{j}\right) .
$$

It follows from (4.23) and (4.26) that

$$
\left|\hat{\mu}_{k, \mathbf{\Phi}_{3}}(\xi)-\hat{\mu}_{k, \mathbf{\Phi}_{4}}(\xi)\right| \leq C\left(\left|\xi_{3}\right| 2^{J k}\right)
$$

for all $k \in \mathbf{Z}_{-},|k|>N$, where $C$ is independent of $k, \rho, \xi$ and $y$.

From (4.25) and (4.27) we see that $\boldsymbol{\Phi}_{3}$ has been reduced to $\boldsymbol{\Phi}_{4}$, whose $L_{p}$ estimate can be derived easily from a result in [23, pages 476-478, where the $L^{p}$ estimate is independent of the coefficients of the polynomials of $t$.

This ends the proof of Theorem 4.7.

Proof of Theorem 1.2. From Lemma 4.2, we may assume that $\Omega$ is a regular atom. For $k \in \mathbf{Z}_{-}$, let $\sigma_{k, \Psi}$ and $\mu_{k, \boldsymbol{\Psi}}$ be defined by (2.1) and (2.2). Since $U$ is bounded and the kernel $K \in L^{1}$ on $U \backslash B_{2}^{1}$, from the definition (1.3) of $T_{\boldsymbol{\Psi}}$, we may assume that $T_{\boldsymbol{\Psi}} f=\sum_{-\infty}^{0} \sigma_{k} * f$. In the proof of Theorem 4.7, after finitely many steps), we reduced the mapping $\boldsymbol{\Psi}$ to $\boldsymbol{\Psi}_{3}$, where in the radial case, $\boldsymbol{\Psi}_{3}=(0, t, \psi(t))$ and in the nonradial case, $\boldsymbol{\Psi}_{3}=\left(0, t, h_{2}(t)\right)$. Since in both cases $\boldsymbol{\Psi}_{3}$ depends only on the radial variable $t$, we see that $\sigma_{k, \Psi_{3}}=0$ since $\int_{S^{1}} \Omega(w) d w=0$. Without loss of generality, we may assume that we reduced $\boldsymbol{\Psi}$ to $\boldsymbol{\Psi}_{3}$ by three steps (in the radial case, exactly three steps). For later convenience we denote $\boldsymbol{\Psi}$ as $\boldsymbol{\Psi}_{0}$. Also, we remark that all the estimates obtained in the proof of Theorem 4.7 for $\mu_{k}$ are valid for $\sigma_{k}$. As indicated for the radial case (see (4.9) and (4.10)), for $i=0,1,2$, one can find $a_{i}>1,0<\alpha_{i}<1$ and linear transformations $T_{i}: R^{3} \rightarrow R^{3}$ such that

$$
\begin{gathered}
\left|\hat{\sigma}_{k, \boldsymbol{\Psi}_{i}}(\xi)\right| \leq C\left(a_{i}^{k}\left|T_{i} \xi\right|\right)^{-\alpha_{i}}, \\
\left|\hat{\mu}_{k, \boldsymbol{\Psi}_{i}}(\xi)-\hat{\mu}_{k, \boldsymbol{\Psi}_{i+1}}(\xi)\right| \leq C\left(a_{i}^{k}\left|T_{i} \xi\right|\right) .
\end{gathered}
$$


Let $s_{i}=\operatorname{rank}\left(T_{i}\right)$. As in (2.8), there are nonsingular linear transformations $G_{i}: R^{3} \rightarrow R^{3}$ be such that

$$
\left|\pi_{s_{i}}^{3} G_{i} \xi\right| \leq\left|T_{i} \xi\right| \leq\left(3-s_{i}+1\right)\left|\pi_{s_{i}}^{3} G_{i} \xi\right|
$$

for every $\xi \in \mathbf{R}^{3}$.

Let $S\left(\mathbf{R}^{s_{i}}\right)$ be the Schwartz class functions in $\mathbf{R}^{s_{i}}$. We choose and fix a function $\varphi_{i} \in C_{0}^{\infty}\left(\mathbf{R}^{s_{i}}\right)$ such that $\varphi_{i}(t) \equiv 1$ for $|t| \leq 1 / 2$, and $\varphi_{i}(t) \equiv 0$ for $|t| \geq 1$. Let $\Phi_{i} \in \mathcal{S}\left(\mathbf{R}^{s_{i}}\right)$ such that $\hat{\Phi}_{i}=\varphi_{i}$.

For $k \in \mathbf{Z}_{-}$and $i=0,1,2$, we define the measures $\tau_{k, i}$ on $\mathbf{R}^{3}$ by

$$
\hat{\tau}_{k, 0}(\xi)=\hat{\sigma}_{k, \mathbf{\Psi}_{0}}(\xi)-\phi_{0}\left(a_{0}^{k} \pi_{s_{0}}^{3} G_{0} \xi\right) \hat{\sigma}_{k, \boldsymbol{\Psi}_{1}}(\xi)
$$

$$
\hat{\tau}_{k, 1}(\xi)=\phi_{0}\left(a_{0}^{k} \pi_{s_{0}}^{3} G_{0} \xi\right) \hat{\sigma}_{k, \mathbf{\Psi}_{1}}(\xi)-\phi_{0}\left(a_{0}^{k} \pi_{s_{0}}^{3} G_{0} \xi\right) \phi_{1}\left(a_{1}^{k} \pi_{s_{1}}^{3} G_{1} \xi\right) \hat{\sigma}_{k, \mathbf{\Psi}_{2}}(\xi),
$$

$$
\begin{aligned}
\hat{\tau}_{k, 2}(\xi) & =\phi_{0}\left(a_{0}^{k} \pi_{s_{0}}^{3} G_{0} \xi\right) \phi_{1}\left(a_{1}^{k} \pi_{s_{1}}^{3} G_{1} \xi\right) \phi_{2} \hat{\sigma}_{k, \boldsymbol{\Psi}_{2}}(\xi) \\
& -\phi_{0}\left(a_{0}^{k} \pi_{s_{0}}^{3} G_{0} \xi\right) \phi_{1}\left(a_{1}^{k} \pi_{s_{1}}^{3} G_{1} \xi\right) \phi_{2}\left(a_{2}^{k} \pi_{s_{2}}^{3} G_{2} \xi\right) \hat{\sigma}_{k, \boldsymbol{\Psi}_{3}}(\xi) .
\end{aligned}
$$

From (4.28) to (4.33) and the choice of $\phi_{i}(t)$, for $i=0,1,2$, we have

$$
\left|\hat{\tau}_{k, i}(\xi)\right| \leq C\left[\min \left\{a_{i}^{k}\left|T_{i} \xi\right|,\left(a_{i}^{k}\left|T_{i} \xi\right|\right)^{-1}\right\}\right]^{\alpha_{i}} .
$$

The proof of Theorem 4.7 yields that for each $i$ we have

$$
\left\|\sigma_{\Psi_{i}}^{*} f\right\|_{p} \leq C_{p}\|f\|_{p}, \quad 1<p<\infty .
$$

Repeating the argument contained in the proof of Lemma $2.1((2.12)-(2.19))$ $(i+1)$ times for $\tau_{i}^{*}$, we see that

$$
\left\|\tau_{i}^{*} f\right\|_{p} \leq C_{p}\|f\|_{p}, \quad 1<p<\infty .
$$

It follows from (4.34) and (4.36) that one can apply Lemma 4.3 for $\tau_{k, i}$ to get

$$
\left\|\sum_{-\infty}^{0} \tau_{k, i} * f\right\|_{p} \leq C_{p}\|f\|_{p}, \quad 1<p<\infty .
$$

Now the inequality (1.8) in Theorem 1.2 is derived from (4.37) since $\sigma_{k, \boldsymbol{\Psi}}=$ $\tau_{k, 0}+\tau_{k, 1}+\tau_{k, 2}$. Here we used the fact that $\sigma_{k, \mathbf{\Psi}_{3}}=0$ for all $k \in \mathbf{Z}_{-}$.

This is the end of the proof of Theorem 1.2.

\section{REFERENCES}

[1] Christ, M., Hilbert transforms along curves: I. Nilpotent groups, Ann. of Math. 122 (1985), 575-596. MR 87f:42039a

[2] Christ, M., Nagel, A., Stein, E. M. and Wainger, S., Singular and Maximal Radon Transforms: Analysis and Geometry, Ann. of Math. 150 (1999), 489-577. MR 2000j:42023

[3] Coifman, R. and Weiss, G., Extension of Hardy spaces and their use in analysis, Bull. Amer. Math. Soc. 83 (1977), 569-645. MR 56:6264

[4] Colzani, L., Hardy spaces on spheres, Ph.D. Thesis, Washington University, St. Louis, 1982.

[5] Connett, W. C., Singular integrals near $L^{1}$, Proc. Symposia Pure Math., 35 (1979), (S. Wainger and G. Weiss eds.), American Mathematical Society, Providence, RI, pp. 163-165. MR 80i:42014

[6] Duoandikoetxea, J. and Rubio de Francia, J. L., Maximal and singular integral operators via Fourier transform estimates, Invent. Math. 84 (1986), 541-561. MR 87f:42046 
[7] Fan, D., Restriction theorems related to atoms, Illinois Jour. Math. 40 (1996), 13-20. MR 97j:42004

[8] Fefferman, R., A note on singular integrals, Proc. Amer. Math. Soc. 74 (1979), 266-270. MR 81e:42025

[9] Fan, D. and Pan, Y., Singular integral operators with rough kernels supported by subvarieties, Amer. J. Math. 119 (1997), 799-839. MR 99c:42029

[10] Fan, D., Guo, K. and Pan, Y., Singular integrals with rough kernels along real-analytic submanifolds in $\mathbf{R}^{n}$, Integral Equations Oper. Theory 33 (1999), 8-19. MR 99k:42031

[11] R. Hardt, Slicing and intersection theory for chains associated with real analytic varieties, Acta Math. 129 (1972), 75-136. MR 47:4110

[12] Hörmander, L., The analysis of linear partial differential operators, I, Springer-Verlag, Berlin, 1983. MR 85g:35002a

[13] Müller, D., Singular kernels supported on homogeneous submanifolds, J. Reine Angew. Math. 356 (1985), 90-118. MR 86i:43014

[14] Namazi, J., A singular integral, Proc. Amer. Math. Soc. 96 (1986), 421-424. MR 87e: 42023

[15] Pan, Y., Boundedness of oscillatory singular integrals on Hardy spaces: II, Indiana Univ. Math. J. 41 (1992), 279-293. MR 97k:42032

[16] Phong, D. H. and Stein, E. M., Singular integrals related to the Radon transform and boundary value problems, Proc. Nat. Acad. Sci. U.S.A. 80 (1983), 7697-7701. MR 86j: 42024

[17] Phong, D. H. and Stein, E. M., Hilbert integrals, singular integrals, and Radon transforms I., Acta Math. 157 (1986), 99-157. MR 88i:42028a

[18] Ricci, F. and Stein, E. M., Harmonic analysis on nilpotent groups and singular integrals I: Oscillatory integrals, Jour. Funct. Anal. 73 (1987), 179-194. MR 88g:42023

[19] Ricci, F. and Stein, E. M., Harmonic analysis on nilpotent groups and singular integrals II: Singular kernels supported on submanifolds, Jour. Funct. Anal. 78 (1988), 56-84. MR 89g:42030

[20] Ricci, F. and Weiss, G., A characterization of $H^{1}\left(\Sigma_{n-1}\right)$, Proc. Symposia Pure Math., 35 (1979), (S. Wainger and G. Weiss eds.), American Mathematical Society, Providence, RI, pp. 289-294. MR 80m:30043

[21] Stein, E. M., Problems in Harmonic Analysis Related to Curvature and Oscillatory Integrals, Proc. Internat. Cong. Math., Berkeley (1986), 196-221. MR 89d:42028

[22] Stein, E. M., Oscillatory integrals in Fourier analysis, Beijing Lectures in Harmonic Analysis, Princeton Univ. Press, Princeton, NJ, 1986, pp. 307-355. MR 88g:42022

[23] Stein, E. M., Harmonic Analysis: Real-Variable Methods, Orthogonality and Oscillatory Integrals, Princeton University Press, Princeton, NJ, 1993. MR 95c:42002

[24] Stein, E. M. and Wainger, S., Problems in harmonic analysis related to curvature, Bull. Amer. Math. Soc. 84 (1978), 1239-1295. MR 80k:42023

Department of Mathematical Sciences, University of Wisconsin-Milwaukee, MilwauKEE, Wisconsin 53201

E-mail address: fan@csd4.csd.uwm.edu

Department of Mathematics, Southwest Missouri State University, Springfield, MisSOURI 65804

E-mail address: kag026f@smsu.edu

Department of Mathematics, University of Pittsburgh, Pittsburgh, Pennsylvania 15260

E-mail address: yibiao+@pitt.edu 